\title{
Solving the muon g-2 anomaly in CMSSM extension with non-universal gaugino masses
}

\author{
Fei Wang, ${ }^{b}$ Kun Wang, ${ }^{a}$ Jin Min Yang ${ }^{c, d, e}$ and Jingya Zhu ${ }^{a, f}$ \\ ${ }^{a}$ Center for Theoretical Physics, School of Physics and Technology, Wuhan University, \\ Wuhan 430072, P.R. China \\ ${ }^{b}$ School of Physics, Zhengzhou University, ZhengZhou 450000, P.R. China \\ ${ }^{c}$ CAS Key Laboratory of Theoretical Physics, Institute of Theoretical Physics, \\ Chinese Academy of Sciences, Beijing 100190, P.R. China \\ ${ }^{d}$ School of Physical Sciences, University of Chinese Academy of Sciences, \\ Beijing 100049, P.R. China \\ ${ }^{e}$ Department of Physics, Tohoku University, Sendai 980-8578, Japan \\ ${ }^{f}$ Enrico Fermi Institute, University of Chicago, Chicago, IL 60637, U.S.A. \\ E-mail: feiwang@zzu.edu.cn, wk2016@whu.edu.cn, jmyang@itp.ac.cn, \\ zhujy@whu.edu.cn
}

AbSTRACT: We propose to generate non-universal gaugino masses in SU(5) Grand Unified Theory (GUT) with the generalized Planck-scale mediation SUSY breaking mechanism, in which the non-universality arises from proper wavefunction normalization with lowest component VEVs of various high dimensional representations of the Higgs fields of SU(5) and an unique F-term VEV by the singlet. Different predictions on gaugino mass ratios with respect to widely studied scenarios are given. The gluino-SUGRA-like scenario, where gluinos are much heavier than winos, bino and universal scalar masses, can be easily realized with appropriate combinations of such high-representation Higgs fields. With six GUTscale free parameters in our scenario, we can solve elegantly the tension between mSUGRA and the present experimental results, including the muon $\mathrm{g}-2$, the dark matter (DM) relic density and the direct sparticle search bounds from the LHC. Taking into account the current constraints in our numerical scan, we have the following observations: (i) The large$\tan \beta(\gtrsim 35)$ samples with a moderate $M_{3}(\sim 5 \mathrm{TeV})$, a small $\left|A_{0} / M_{3}\right|(\lesssim 0.4)$ and a small $m_{A}(\lesssim 4 \mathrm{TeV})$ are favoured to generate a $125 \mathrm{GeV}$ SM-like Higgs and predict a large muon g-2, while the stop mass and $\mu$ parameter, mainly determined by $\left|M_{3}\right|\left(\gg M_{0},\left|M_{1}\right|,\left|M_{2}\right|\right)$, can be about $6 \mathrm{TeV}$; (ii) The moderate-tan $\beta$ (35 40) samples with a negative $M_{3}$ can have a light smuon $(250 \sim 450 \mathrm{GeV})$ but a heavy stau $(\gtrsim 1 \mathrm{TeV})$, which predict a large muon g-2 but a small $\operatorname{Br}\left(B_{s} \rightarrow \mu^{+} \mu^{-}\right)$; (iii) To obtain the right DM relic density, the annihilation 
mechanisms should be stau exchange, stau coannihilation, chargino coannihilation, slepton annihilation and the combination of two or three of them; (iv) To obtain the right DM relic density, the spin-independent DM-nucleon cross section is typically much smaller than the present limits of XENON1T 2018 and also an order of magnitude lower than the future detection sensitivity of LZ and XENONnT experiments.

Keywords: Supersymmetry Phenomenology

ARXiv EPrint: 1808.10851 


\section{Contents}

1 Introduction 1

2 Generating non-universal gaugino masses in SU(5) 2

$\begin{array}{lll}3 & \text { The scan and constraints } & 6\end{array}$

4 Numerical results and discussions $\quad 8$

$\begin{array}{lll}5 & \text { Summary and conclusions } & 15\end{array}$

A Correlations between the parameters at SUSY scale and GUT scale $\quad 16$

B The detail information of the 7 benchmark points 20

\section{Introduction}

Low energy supersymmetry (SUSY), which is well motivated to solve the hierarchy problem, is one of the most appealing new physics candidates beyond the Standard Model (SM). The gauge coupling unification, which cannot be realized in the SM, can be successfully realized in the framework of weak scale SUSY. Besides, assuming R-parity conservation, the lightest SUSY particle (LSP) can be a promising dark matter (DM) candidate with the right DM relic density.

However, there are over 100 physical free parameters in the minimal SUSY model (MSSM), including the soft masses, phases and mixing angles that cannot be rotated away by redefining the phases and flavor basis for the quark and lepton supermultiplets. In practice, some universalities of certain soft SUSY breaking parameters as high scale inputs are usually adopted. In the constrained MSSM (CMSSM), the gaugino masses, the sfermion masses and the trilinear couplings are all assumed to be universal at the GUT scale, respectively. Thus, CMSSM only has five free parameters, i.e., $\tan \beta, M_{0}, A_{0}, M_{1 / 2}$ and the sign of $\mu$. All the low energy soft SUSY breaking parameters can be determined by these five inputs through the renormalization group equations (RGEs) running from the GUT scale to the EW scale.

So far the null search results of the gluino and the first two generations of squarks together with the $125 \mathrm{GeV}$ Higgs discovery $[1,2]$ at the LHC have severely constrained the parameter space of CMSSM. For example, to provide a $125 \mathrm{GeV}$ SM-like Higgs, the stop masses should be around $10 \mathrm{TeV}$ or the trilinear stop mixing parameter $A_{t}$ should be quite large. Besides, in order for the gluino to escape the LHC bounds, the universal gaugino mass at the GUT scale $\left|M_{1 / 2}\right|$ should be larger than about $1 \mathrm{TeV}\left(m_{\tilde{g}} \simeq 2\left|M_{1 / 2}\right|\right.$ ), and thus the bino-like neutralino is bounded to be higher than about $400 \mathrm{GeV}$. All 
the electroweakinos (higgsinos, sleptons, sneutrinos) are all bounded to be heavier than several hundreds of GeV in CMSSM, and hence the SUSY contributions to the muon g-2 cannot be large enough to account for the discrepancy reported by the Brookhaven AGS [3-6]. In fact, CMSSM was found to be excluded at $90 \%$ confidence level [7] if it is required to account for both the muon g-2 anomaly and the recent LHC constraints on SUSY particles. The neutralino dark matter in CMSSM, which is heavier than several hundreds of $\mathrm{GeV}$, can mainly have four annihilation mechanisms: stau coannihilation, stop coannihilation, A/H funnel, hybrid (note that the $\mathrm{h} / \mathrm{Z}$ funnel cannot happen and the focus point or $\chi_{1}^{ \pm}$coannihilation is severely constrained by the recent dark matter direct detection limits) $[6,8]$. We should mention that even if only the DM relic density upper bound is considered in addition to the muon g-2, a global fit by the GAMBIT [9] collaboration still disfavors the CMSSM.

Motivated by the tension between CMSSM and the experiment results, several extensions of CMSSM have been proposed, such as introducing right-handed neutrinos [10-12], singlet scalars [13-18] or vector-like supermultiplets $[19,20]$. Other proposals were also considered, such as relaxing universal conditions at the GUT scale [12, 21-28] or reducing the universal conditions to certain sub-GUT scale [29-31] (such as the 'mirage' unification scale [32]) or including the reheating temperature in the early universe as an extra parameter [33]. Among these approaches, the non-universal gaugino mass (NUGM) [34-37] scenarios are well motivated both theoretically and phenomenologically, which can be realized by some special structure of gauge kinetic function in string models [38-41] or the GUT [42].

In this work, we propose a NUGM extension of mSUGRA, where the non-universality arises from proper wavefunction normalization with lowest component VEVs of various high dimensional representations of the Higgs fields of SU(5) and an unique F-term VEV by the singlet. By properly choosing the parameters, we can generate the gaugino hierarchy $M_{3} \gg M_{1}, M_{2}$ and obtain a low energy spectrum which can escape the LHC mass bounds for colored sparticles and at the same time give the $125 \mathrm{GeV}$ Higgs mass. The muon g-2 anomaly can also be solved with $M_{0}$ at a few hundreds of GeV. Besides, the flavour bounds, the LHC direct search bounds as well as the updated dark matter constraints can all be satisfied. Note that this setting can be fitted into the $\tilde{g}$ SUGRA scenario proposed in [43].

This paper is organized as follows. We propose our approach to generate non-universal gaugino masses in SU(5) GUT with the generalized Planck-scale mediation of SUSY breaking in section 2. In section 3, we discuss the constraints on the model. In section 4 , we present our numerical results and give some discussions. Finally, we draw our conclusions in section 5 .

\section{Generating non-universal gaugino masses in $\mathrm{SU}(5)$}

As a low energy phenomenological model, the MSSM is not very predictive because it has more than one hundred free parameters. The gaugino mass hierarchy, which is also not specified in MSSM, is very important in understanding the nature of dark matter. For example, possible signals for neutralino dark matter direct detection experiments depend on the ratio of each component of the dark matter particle. So it is desirable to seek for 
some UV-completed models that can predict the low energy soft SUSY breaking parameters with few input parameters.

Some UV-completed models of MSSM, e.g., the gravity mediated SUSY breaking scenarios with the simplest Kahler potential, predict universal gaugino masses at the GUT scale. After RGE running to EW scale, the approximate ratio for gaugino masses ${ }^{1}$ is $M_{1}: M_{2}: M_{3} \approx 1: 2: 6$. We know that the latest LHC results have pushed the gluino mass to about $2 \mathrm{TeV}$, and thus the neutral electroweakinos are also heavy and cannot solve the muon $g-2$ anomaly. Actually, the gaugino masses at the GUT scale are not necessarily universal. In realistic SUSY GUT models, certain high dimensional GUT group representations of Higgs fields may play an essential role in solving the doublet-triplet splitting problem or generating realistic fermion ratios if Yukawa unification is further assumed. With such high dimensional Higgs fields, the universal soft SUSY breaking masses can receive additional non-universal parts. For example, the scenarios with non-universal gaugino masses have been studied in $[27,44-62]$ and references therein. General results of soft SUSY breaking parameters in the generalized SUGRA [63-65] for SU(5), SO(10) and E6 GUT models involving various high dimensional Higgs fields with different symmetry breaking chains have been discussed in $[63,64,66]$. Some applications have been also studied [67-70].

The gaugino masses can always be given by the following non-renomalizable superpotential terms

$$
W \supseteq \frac{f_{a}}{4}\left[W^{a} W^{a}+a_{1} \frac{T}{\Lambda} W^{a} W^{a}+b_{1} \frac{1}{\Lambda} W^{a} \Phi_{a b} W^{b}+c_{1} \frac{T}{\Lambda^{2}} W^{a} \Phi_{a b} W^{b}\right],
$$

with $\Lambda$ being a typical UV energy scale (say the Planck scale $M_{P l}$ ) upon the GUT scale. The chiral superfield $T$ is a GUT group singlet and $\Phi_{a b}$ is a chiral superfield lying in any of the irreducible representations within the symmetric group production decomposition of adjoint representations. For example, in the framework of SU(5) GUT, $\Phi_{a b}$ can belong to

$$
(\mathbf{2 4} \otimes \mathbf{2 4})_{\mathrm{symm}}=\mathbf{1} \oplus \mathbf{2 4} \oplus \mathbf{7 5} \oplus \mathbf{2 0 0} .
$$

After $\Phi_{a b}$ or $T$ acquiring an F-term VEVs, soft SUSY breaking gaugino masses will be predicted. For example, the term proportional to $a_{1}$ will generate universal gaugino masses with non-vanishing $\left\langle F_{T}\right\rangle$, while the term proportional to $b_{1}$ will generate non-universal gaugino parts with non-vanishing $\left\langle F_{\Phi_{a b}}\right\rangle$. In most of the previous studies, non-vanishing $\mathrm{F}-$ term VEVs of the GUT non-singlet Higgs field $\Phi_{a b}$ are necessarily present to generate nonuniversal gaugino masses. In principle, the soft sfermion masses or trilinear couplings may also receive additional non-universal contributions from such high dimensional operators.

Although it is indeed possible to realize SUSY breaking with a F-term VEV for a gauge non-singlet superfield through model buildings, for example in typical dynamical SUSY breaking models or ISS-type models, it is more natural to realize SUSY breaking with a gauge singlet F-term VEV. We propose a new approach in which the leading nonuniversality of gaugino masses comes from the wavefunction normalization with a F-term

\footnotetext{
${ }^{1}$ Such a ratio at the EW scale is also predicted by the minimal gauge mediated SUSY breaking mechanism.
} 
VEV for a gauge singlet only. ${ }^{2}$ Although simple, this possibility, which leads to different predictions on gaugino hierarchy, has not been emphasized and discussed in previous nonuniversal gaugino masses literatures.

We can consider the most general combination involving the 24, 75 and 200 representations of Higgs fields of SU(5) GUT group and the gauge singlet $T$

$$
\mathcal{L}=\int d^{2} \theta\left(\frac{f_{a}}{4} W^{a} W^{b}\right) \frac{1}{\Lambda}\left[c_{0} T \delta_{a b}+c_{1}\left(H_{\mathbf{2 4}}\right)_{a b}+c_{2}\left(H_{\mathbf{7 5}}\right)_{a b}+c_{3}\left(H_{\mathbf{2 0 0}}\right)_{a b}\right] .
$$

In previous studies on non-universal gaugino masses, as noted previously, non-vanishing $F_{H_{r}}$ are necessarily present to generate non-universal gaugino masses with (almost) trivial kinetic terms for gauginos. It is however possible that only the GUT group singlet $T$ acquires both F-term VEV $\left\langle F_{T}\right\rangle$ and lowest component VEV $\langle T\rangle$ while all other high dimensional representation Higgs fields acquire only the lowest component VEVs that still preserve the $\mathrm{SU}(3)_{C} \times \mathrm{SU}(2)_{L} \times \mathrm{U}(1)_{Y}$ gauge group

$$
\langle T\rangle=T_{0}+\theta^{2} F_{T}, \quad\left\langle H_{a b}^{r}\right\rangle=v_{U} M_{a b}^{r}
$$

with $M_{a b}^{r}$ being the group factor for the representation $r$ and $v_{U}$ the GUT breaking scale which is assumed to be independent of the SU(5) representation and universal for all $H_{a b}^{r}$. The VEVs of the Higgs field $\Phi_{\mathbf{2 4}}$ in the adjoint representation can be expressed as a $5 \times 5$ matrix

$$
\left\langle\Phi_{\mathbf{2 4}}\right\rangle=v_{U} \sqrt{\frac{3}{5}} \operatorname{diag}\left(-\frac{1}{3},-\frac{1}{3},-\frac{1}{3}, \frac{1}{2}, \frac{1}{2}\right),
$$

while the VEVs of the Higgs field $\Phi_{\mathbf{7 5}}$ can be expressed as a $10 \times 10$ matrix

$$
\left\langle\Phi_{\mathbf{7 5}}\right\rangle=\frac{v_{U}}{2 \sqrt{3}} \operatorname{diag}(\underbrace{1, \cdots, 1}_{3}, \underbrace{-1, \cdots,-1}_{6}, 3) .
$$

Similarly, the VEVs of the Higgs field $\Phi_{\mathbf{2 0 0}}$ can be expressed as a $15 \times 15$ matrix

$$
\left\langle\Phi_{\mathbf{2 0 0}}\right\rangle=\frac{v_{U}}{2 \sqrt{3}} \operatorname{diag}(\underbrace{1, \cdots, 1}_{6}, \underbrace{-2, \cdots,-2}_{6}, \underbrace{2, \cdots, 2}_{3}) .
$$

As $T_{0}$ is a GUT group singlet, the VEV $T_{0}$ can be of order $\Lambda$ without spoiling GUT. The kinetic term after substituting the lowest component VEV will take the form

$$
W \supseteq \frac{f_{a}}{4} W^{a} W^{b}\left[\left(1+a_{1} \frac{T}{\Lambda}\right) \delta_{a b}+\sum_{r} c_{i} \frac{v_{U}}{\Lambda}\left\langle M_{a b}^{r}\right\rangle\right] .
$$

As $v_{U} \ll \Lambda$ and $T_{0} \simeq \Lambda$, the term proportional to $\delta_{a b}$ will be the leading normalization factor. If this term nearly vanishes by choosing a proper $a_{1}$, the second term, which is nonuniversal for three gauge couplings, will generate a different wavefunction normalization

\footnotetext{
${ }^{2}$ We should note that it is possible to generate subleading non-universal gaugino masses from the $c_{1}$ term of eq. (2.1) with a suppression factor $\Lambda^{-2}$ in comparsion with the leading universal gaugino mass part which is suppressed by $\Lambda^{-1}$.
} 
factor. On the other hand, substituting the F-term VEV $F_{T}$ will generate universal gaugino masses for non-canonical gauge fields. After normalizing the gauge kinetic term to canonical form, non-universal gaugino masses will be generated. The prediction in this scenario is different from previous studies. In table 1, we list our prediction for gaugino mass ratios in different $\mathrm{SU}(5)$ representations, in comparison with previous studies (e.g., ref. [62]). For example, if only the $\mathbf{2 4}$ representation Higgs is present other than $T$, the gaugino ratio is given by

$$
M_{1}: M_{2}: M_{3}=1: \frac{1}{3}:-\frac{1}{2},
$$

at the GUT scale which will predict the gaugino ratio $3: 2:-9$ at the EW scale. So the wino is the lightest gaugino and possibly be the DM candidate in contrary to the widely studied scenarios with bino as the lightest gaugino for $\left\langle F_{\mathbf{2 4}}\right\rangle \neq 0$. Another example, although we adopt the most general form of combinations, gluino SUGRA can in fact be realized with only one 200 or 75 representation, in which the gluino can be much (almost $5 \sim 10$ times) heavier than wino and bino at EW scale. For the most general combinations involving all 24, 75 and 200 Higgs fields, we can obtain the gaugino ratio $M_{1}: M_{2}: M_{3}$ as

$$
\left[-\frac{c_{1}}{4 \sqrt{15}}+\frac{5 c_{2}}{4 \sqrt{3}}+\frac{5 c_{3}}{2 \sqrt{3}}\right]^{-1}:\left[-\frac{3 c_{1}}{4 \sqrt{15}}-\frac{3 c_{2}}{4 \sqrt{3}}+\frac{c_{3}}{2 \sqrt{3}}\right]^{-1}:\left[\frac{c_{1}}{2 \sqrt{15}}-\frac{c_{2}}{4 \sqrt{3}}+\frac{c_{3}}{4 \sqrt{3}}\right]^{-1},
$$

at the GUT scale. So we can see that one can get an arbitrary gaugino ratio at GUT scale with different choices of $c_{i}$. This result is different from the widely studied scenarios in which both the high-representation Higgs fields $H_{a b}$ and $T$ acquire universal F-term VEVs $F_{U}$ with trivial kinetic terms, which gives the gaugino mass ratio $M_{1}: M_{2}: M_{3}$ at the GUT scale as

$$
\left[c_{0}-\frac{c_{1}}{4 \sqrt{15}}+\frac{5 c_{2}}{4 \sqrt{3}}+\frac{5 c_{3}}{2 \sqrt{3}}\right]:\left[c_{0}-\frac{3 c_{1}}{4 \sqrt{15}}-\frac{3 c_{2}}{4 \sqrt{3}}+\frac{c_{3}}{2 \sqrt{3}}\right]:\left[c_{0}+\frac{c_{1}}{2 \sqrt{15}}-\frac{c_{2}}{4 \sqrt{3}}+\frac{c_{3}}{4 \sqrt{3}}\right] .
$$

So, an arbitrary gaugino ratio of $M_{1}: M_{2}: M_{3}$ at the GUT scale can be obtained with properly chosen coefficients $c_{1}, c_{2}, c_{3}$ or $c_{0}, c_{1}, c_{2}, c_{3}$. Besides, the new and old approaches will in general lead to different predictions on the nature of lightest gaugino.

An interesting region will appear if $M_{3} \gg M_{2}, M_{1}$. In this region, the colored sfermions are heavy even for a very small $M_{0}$ (which is the universal sfermion mass parameter) because of the loop corrections involving a heavy $M_{3}$. The non-colored sfermions will, however, still be light if the GUT scale mass $M_{1,2} \ll M_{3}$. This region, which is called the gluino-SUGRA region [43], is well motivated to solve the muon $g-2$ anomaly [71] and at the same time be consistent with the LHC predictions. In our new approach, the gluinoSUGRA region is easily realized if the denominators of the third term within eq. (2.10) nearly vanishes

$$
\frac{c_{1}}{2 \sqrt{15}}-\frac{c_{2}}{4 \sqrt{3}}+\frac{c_{3}}{4 \sqrt{3}} \approx 0
$$




\begin{tabular}{|c|c|c|}
\hline representations & gaugino ratios in our work & gaugino ratios in previous studies \\
\hline 1 & GUT $1: 1: 1, \quad$ EW $1: 2: 6$ & GUT $1: 1: 1, \quad$ EW $1: 2: 6$ \\
\hline 24 & GUT $1: \frac{1}{3}:-\frac{1}{2}, \quad$ EW $3: 2:-9$ & GUT $1: 3:-2, \quad$ EW $1: 6:-12$ \\
\hline 75 & GUT $-\frac{1}{5}: \frac{1}{3}: 1, \quad$ EW $-3: 10: 90$ & GUT $-5: 3: 1, \quad$ EW $-5: 6: 6$ \\
\hline 200 & GUT $\frac{1}{10}: \frac{1}{2}: 1, \quad$ EW $1: 10: 60$ & GUT $10: 2: 1$ \\
\hline
\end{tabular}

Table 1. Comparison of our predictions with previous studies (e.g., ref. [62]) on gaugino mass ratios in different $\mathrm{SU}(5)$ representations, where 'GUT' is for results at GUT scale and ' $\mathrm{EW}$ ' is for results at $\mathrm{EW}$ scale.

while the denominators of the first two terms are non-vanishing. In the widely studied approach which is given by eq. (2.11), to realize the gluino-SUGRA region, the first two terms within the second line of eq. (2.11) need to vanish approximately while the third term should be non-vanishing. Solving for $c_{1}, c_{2}$ in terms of $c_{0}$ and $c_{3}$ gives

$$
c_{1}=\frac{20 \sqrt{5}}{9} c_{3}+\frac{16 \sqrt{5}}{9} c_{0}, c_{2}=-\frac{14}{9} c_{3}-\frac{4 \sqrt{3}}{9} c_{0}
$$

where $c_{3} \neq-\frac{8 \sqrt{3}}{7} c_{0}$.

\section{The scan and constraints}

In order to illustrate the salient features of our scenarios, we scan the six dimensional parameter space considering all current experimental constraints. The package NMSSMTools $[72,73]$ is used in our numerical scan to obtain the low energy SUSY spectrum. We know that in case $\lambda \sim \kappa \rightarrow 0$ and $A_{\lambda}$ is small, the singlet superfield within the NMSSM will decouple from other superfields and the NMSSM will reduce to the MSSM plus a decoupled heavy singlet scalar and singlino. So the MSSM spectrum can be calculated with NMSSMTools. In our scan, we use the program NMSPEC_MCMC [74] in NMSSMTools_5.2.0 [72, 73]. The ranges of parameters in our scan are

$$
\begin{aligned}
1 \mathrm{TeV}<\left|M_{3}\right| & <10 \mathrm{TeV}, & \left|A_{0}\right| & <20 \mathrm{TeV}, \\
M_{0},\left|M_{1}\right|,\left|M_{2}\right| & <1 \mathrm{TeV}, & 1 & <\tan \beta<60,
\end{aligned}
$$

where we choose a large $\left|M_{3}\right|$ to escape the LHC bounds on colored sparticles and a large $\left|A_{0}\right|$ to generate the $125 \mathrm{GeV}$ Higgs mass. Small $M_{0},\left|M_{1}\right|,\left|M_{2}\right|$ and a large $\tan \beta$ are chosen to give large SUSY contributions to the muon g-2 and a low mass for dark matter particle.

In our scan, we consider the following constraints:

(1) The theoretical constraints of vacuum stability, and no Landau pole below $M_{\mathrm{GUT}}[72-74]$.

(2) The lightest CP-even Higgs boson $h$ as the SM-like Higgs boson with a mass of $123-127 \mathrm{GeV}$. The SM-like Higgs mass is calculated including corrections of top/bottom sector at full 1 loop and leading logs 2 loop level, and other sfermions, 
charginos/neutralinos, and gauge bosons at leading logs 1 loop level. ${ }^{3}$ Its production rates should fit the LHC data globally $[123,124]$ with the method in refs. [125, 126].

(3) The searches for low mass and high mass resonances at the LEP, Tevatron, and LHC, which constrained the production rates of heavy Higgs bosons. We implement these constraints by the package HiggsBounds-5.1.1beta [127].

(4) The constraints for squarks and gluino from the LHC:

$$
m_{\tilde{g}} \gtrsim 2 \mathrm{TeV}, \quad m_{\tilde{t}} \gtrsim 0.7 \mathrm{TeV}, \quad m_{\tilde{q}_{1,2}} \gtrsim 2 \mathrm{TeV}
$$

and the lower mass bounds of charginos, sleptons from the LEP:

$$
m_{\tilde{\tau}} \gtrsim 93.2 \mathrm{GeV}, \quad m_{\chi^{ \pm}} \gtrsim 103.5 \mathrm{GeV} .
$$

(5) The searches for chargino $\chi_{1}^{ \pm}$and next-to-lightest neutralino $\chi_{2}^{0}$ at the LHC:

$$
p p \rightarrow \chi_{1}^{+} \chi_{1}^{-}, \chi_{1}^{ \pm} \chi_{2}^{0}
$$

For these searches, we only employ the channels with tau leptons in final states [128] because we checked that the dominated decay channels of $\chi_{1}^{ \pm}$and $\chi_{2}^{0}$ are

$$
\begin{array}{lll}
\chi_{1}^{ \pm} \rightarrow \tilde{\tau}^{ \pm} \nu_{\tau}, & \chi_{2}^{0} \rightarrow \tilde{\tau}^{ \pm} \tau^{\mp} & \left(\text { for } m_{\chi_{1}^{ \pm}}>m_{\tilde{\tau}}\right), \\
\chi_{1}^{ \pm} \rightarrow \tau^{ \pm} \nu_{\tau} \chi_{1}^{0}, & \chi_{2}^{0} \rightarrow \tau^{ \pm} \tau^{\mp} \chi_{1}^{0} & \left(\text { for } m_{\chi_{1}^{ \pm}}<m_{\tilde{\tau}}\right) .
\end{array}
$$

(6) Constraints from B physics, such as $B \rightarrow X_{s} \gamma, B_{s} \rightarrow \mu^{+} \mu^{-}, B_{d} \rightarrow \mu^{+} \mu^{-}$and $B^{+} \rightarrow \tau^{+} \nu_{\tau}$, and the mass differences $\Delta M_{d}, \Delta M_{s}[129-132]$

$$
\begin{aligned}
1.7 \times 10^{-9}<B r\left(B_{s} \rightarrow \mu^{+} \mu^{-}\right) & <4.5 \times 10^{-9}, \\
1.1 \times 10^{-10}<B r\left(B_{d} \rightarrow \mu^{+} \mu^{-}\right) & <7.1 \times 10^{-10}, \\
2.99 \times 10^{-4}<\operatorname{Br}(b \rightarrow s \gamma) & <3.87 \times 10^{-4} .
\end{aligned}
$$

(7) The constraints of muon anomalous magnetic moment (muon g-2) at $2 \sigma$ level. For the experimental data and SM calculation without the Higgs contribution (because there is a SM-like Higgs boson in SUSY models contributing to $\left.\Delta a_{\mu}\right)$, we use $[3,133-136]$

$$
\begin{aligned}
a_{\mu}^{\mathrm{ex}} & =(11659208.0 \pm 6.3) \times 10^{-10} \\
\Delta a_{\mu} & \equiv a_{\mu}^{\mathrm{ex}}-a_{\mu}^{\mathrm{SM}}=(27.4 \pm 9.3) \times 10^{-10} .
\end{aligned}
$$

\footnotetext{
${ }^{3}$ With fixed-order method, full one-loop corrections have been calculated diagrammatically in refs. [7578], dominant two-loop corrections in refs. [79-102] and partial three-loop corrections in refs. [103-105]. Also, effective field theory (EFT) methods were applied in refs. [106-111], and fourth logarithmic order corrections was calculated in ref. [112]. Besides, hybrid methods have also been developed [113-119], and been implemented in the publicly available code FeynHiggs [120-122]. We checked our Higgs masses with FeynHiggs-2.14.3, and found they coincide at $2 \mathrm{GeV}$ level for most samples.
} 

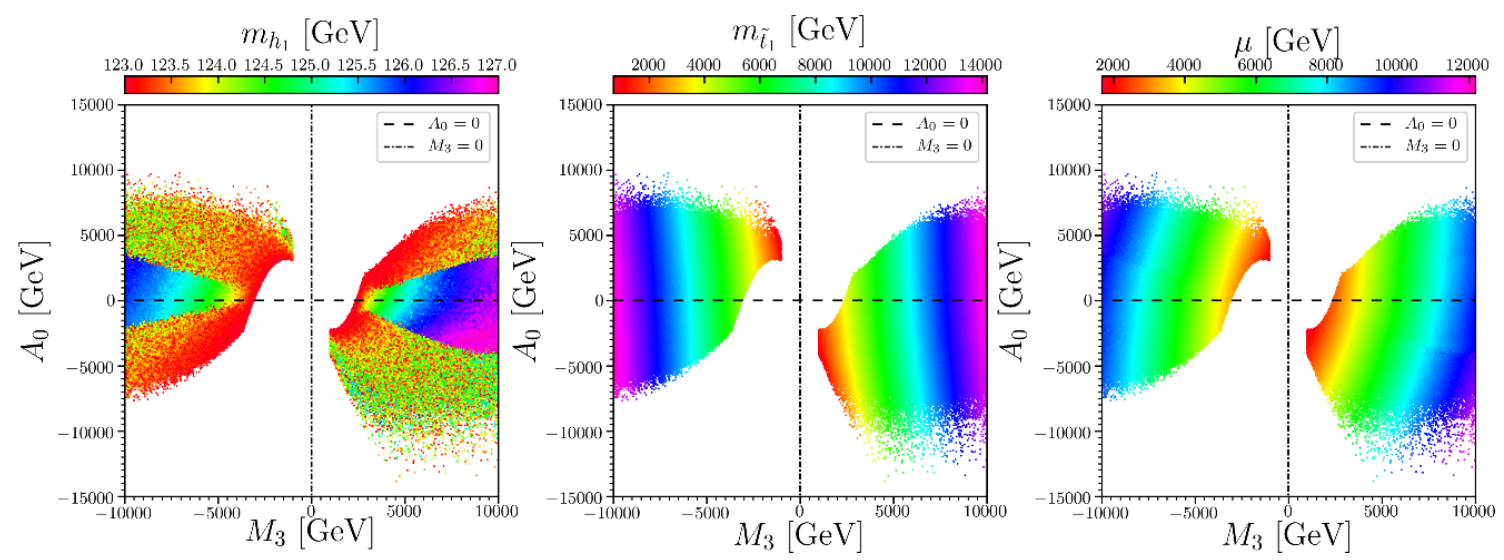

Figure 1. Surviving samples in the $A_{0}$ versus $M_{3}$ planes, with colors indicating the lighter Higgs mass $m_{h}$ (left), lighter stop mass $m_{\tilde{t}_{1}}$ (middle) and higgsino mass parameter $\mu$ (right), respectively. In these 2-dimension planes, larger- $\Delta a_{\mu}$ samples are projected on top of smaller ones.

We calculate the SUSY contribution $\Delta a_{\mu}$ including the SM-like Higgs boson, and require $\Delta a_{\mu}$ to lie at the $2 \sigma$ level. We also include the theoretical uncertainty in SUSY $\Delta a_{\mu}$ calculations, which is about $\delta^{\text {th }} \approx 3 \times 10^{-10}[137]^{4}$

(8) Constraints from dark matter relic density by WMAP/Planck [132, 142, 143], and the 2018 result of direct searches for dark matter at XENON1T [144]. We require the lightest neutralino $\chi_{1}^{0}$ to be the dark matter candidate and calculate its relic density and cross sections by micrOMEGAs [145] inside NMSSMTools. For DM relic density, we only apply the upper bound, e.g., $0 \leq \Omega \leq 0.131$, as other dark matter species may also contribute to the DM relic density [33, 72, 73, 148]. For DM-nucleon scattering cross section, we rescale the original values by $\Omega / \Omega_{0}$ with $\Omega_{0} h^{2}=0.1187$ to impose the XENON1T constraint.

We take a multi-path Markov Chain Monte Carlo (MCMC) scan in the parameter space. In total, we get nearly $10^{7}$ surviving samples.

\section{Numerical results and discussions}

In figure 1 , we project the surviving samples on $A_{0}$ versus $M_{3}$ planes, with colors indicating the SM-like Higgs mass $m_{h}$ (left), the lighter stop mass $m_{\tilde{t}_{1}}$ (middle) and the higgsino mass parameter $\mu$ (right), respectively. In figure 2 , we project the surviving samples in the $A_{0}$ versus $M_{3}$ (left and middle), $\tan \beta$ versus the CP-odd Higgs mass $m_{A}$ (right) planes, with colors indicating $m_{A}$ (left), $\tan \beta$ (middle), and $\operatorname{Br}\left(B_{s} \rightarrow \mu^{+} \mu^{-}\right)$(right), respectively. In all these 2-dimension planes in figure 1 and figure 2 , larger- $\Delta a_{\mu}$ samples are shown on top of smaller ones. From the left plane of figure 1, left and middle planes of figure 2, we can

\footnotetext{
${ }^{4}$ The $\Delta a_{\mu}$ includes chargino [137], neutralino [138], and Higgs [139] contributions, all at 2-loop level. The theoretical uncertainty is calculated as $\delta^{\text {th }} \equiv 2.8 \times 10^{-10}+0.02\left|\Delta a_{\mu}^{1 \mathrm{~L}}\right|+0.3\left|a_{\mu}^{2 \mathrm{~L}}-a_{\mu}^{1 \mathrm{~L}}\right|[137]$, a little larger than that in refs. $[140,141]$. And the theoretical uncertainty is added linearly to $\Delta a_{\mu}$, totally required to satisfy $a_{\mu}^{\mathrm{ex}}-a_{\mu}^{\mathrm{SM}}$ at $2 \sigma$ level.
} 

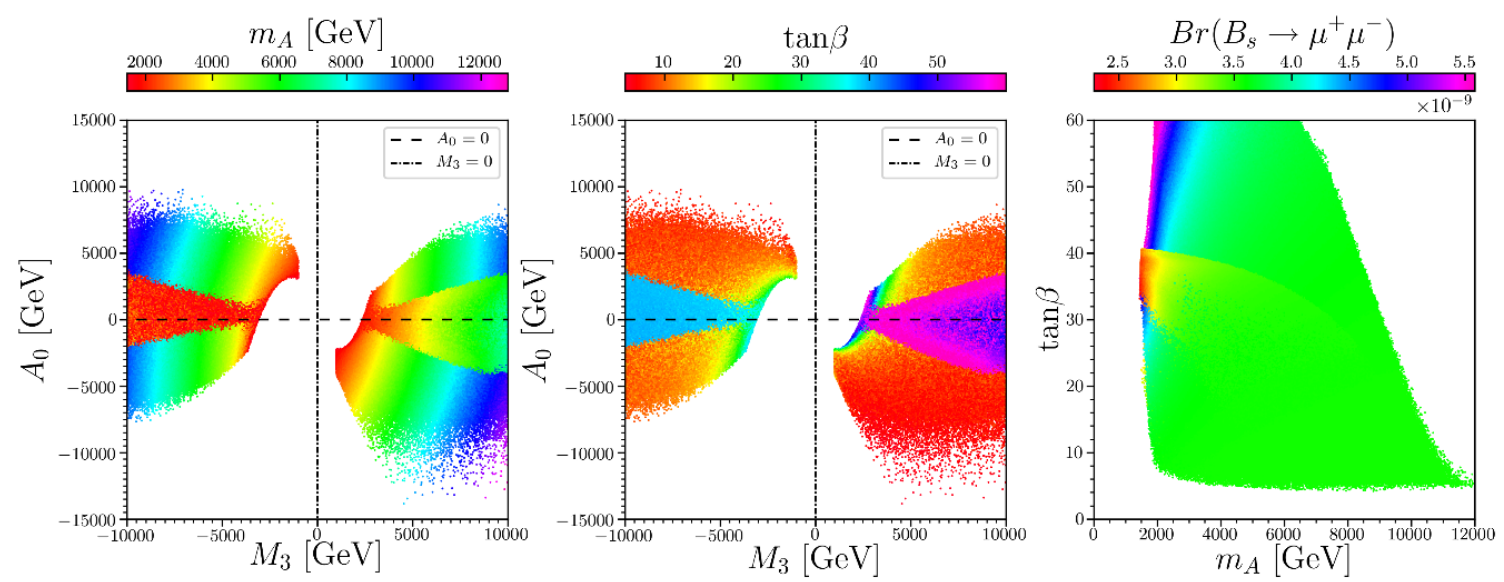

Figure 2. Surviving samples in the $A_{0}$ versus $M_{3}$ (left and middle), $\tan \beta$ versus the CP-odd Higgs mass $m_{A}$ (right) planes. The colors indicate $m_{A}$ (left), $\tan \beta$ (middle), and $\operatorname{Br}\left(B_{s} \rightarrow \mu^{+} \mu^{-}\right.$) (right), respectively. In these 2-dimension planes, larger- $\Delta a_{\mu}$ samples are projected on top of smaller ones.

see that the larger- $\Delta a_{\mu}$ (or topside) samples on the $M_{3}-A_{0}$ planes can be sorted into four classes roughly:

Class A : $40 \lesssim \tan \beta \lesssim 60,\left|A_{0} / M_{3}\right| \lesssim 0.35, M_{3}>0, m_{A} \lesssim 7 \mathrm{TeV}$

Class B : $35 \lesssim \tan \beta \lesssim 40,\left|A_{0} / M_{3}\right| \lesssim 0.35, M_{3}<0, m_{A} \lesssim 3 \mathrm{TeV}$;

Class C : $\tan \beta \gtrsim 15,\left|A_{0} / M_{3}\right| \gtrsim 0.35, m_{A} \lesssim 4 \mathrm{TeV}$;

Class D : $\tan \beta \lesssim 15,\left|A_{0} / M_{3}\right| \gtrsim 0.35, m_{A} \gtrsim 4 \mathrm{TeV}$.

From the middle and right planes of figure 1 , we can see that the lighter stop mass $m_{\tilde{t}_{1}}$ and parameter $\mu$ are mainly determined by $M_{3}$ and $A_{0}$. Since $A_{0}, M_{3} \gg M_{0}, M_{1}, M_{2}$ and $\tan \beta \gg 1$, according to correlations between the parameters at SUSY scale and GUT scale are listed in appendix A, we can have following approximations for most surviving samples:

$$
\begin{aligned}
M_{\tilde{Q}_{3}} & \approx M_{\tilde{U}_{3}} \approx 1.5\left|M_{3}\right|, \\
A_{t} & \approx-1.0 M_{3}-0.4 A_{0}, \\
\mu & =\sqrt{\frac{M_{H_{d}}^{2}-M_{H_{u}}^{2} \tan ^{2} \beta}{\tan ^{2} \beta-1}-\frac{m_{Z}^{2}}{2}} \approx\left|M_{H_{u}}\right| \approx \sqrt{0.91 M_{3}^{2}-0.18 A_{0} M_{3}+0.09 A_{0}^{2}} .
\end{aligned}
$$

Then we have

$$
m_{\tilde{t}_{1,2}} \approx \sqrt{\left(1.5 M_{3}\right)^{2} \mp v\left|X_{t}\right|}
$$

where $v=174 \mathrm{GeV}$ is the Higgs VEV in the SM, and $X_{t} \equiv A_{t}-\mu / \tan \beta$. The SM-like Higgs mass with one-loop correction of stops is given by

$$
\begin{aligned}
m_{h}^{2} & =m_{Z}^{2} \cos ^{2} 2 \beta+\frac{3}{4 \pi^{2}} \frac{m_{t}^{4}}{v^{2}}\left[\log \frac{M_{\tilde{t}}^{2}}{m_{t}^{2}}+\frac{2 X_{t}^{2}}{M_{\tilde{t}}^{2}}\left(1-\frac{X_{t}^{2}}{12 M_{\tilde{t}}^{2}}\right)\right], \\
& \approx m_{Z}^{2}+\frac{3}{4 \pi^{2}} \frac{m_{t}^{4}}{v^{2}} \times\left(2 \log \frac{M_{\tilde{t}}}{m_{t}}+2 x^{2}-\frac{1}{6} x^{4}\right)
\end{aligned}
$$


where $x \equiv\left|X_{t} / M_{\tilde{t}}\right|$ and $M_{\tilde{t}} \equiv \sqrt{m_{\tilde{t}_{1}} m_{\tilde{t}_{2}}}$. For the large-tan $\beta$ samples in Class A and B, with $\tan \beta \gg 1,\left|A_{0} / M_{3}\right| \lesssim 0.4$, and farther approximating in eq. (4.4), we can get the Higgs mass can be mainly determined by $M_{3}$, and when $\left|M_{3}\right| \sim 5 \mathrm{TeV}$ the SM-like Higgs can get to $125 \mathrm{GeV}$ with 1-loop stop corrections. For moderate/large-tan $\beta$ samples in Class $\mathrm{C}$, Higgs mass are mainly determined by both $M_{3}$ and $A_{0}$. While for small-tan $\beta$ samples (Class D), $\tan \beta$ can also play a part in determining the SM-like Higgs mass. These are some of our new findings for larger- $\Delta a_{\mu}$ samples in this work.

Just like the parameter point shown in eqs. (A.14), (A.15) in appendix A, for the samples in Class $\mathrm{C}$ and $\mathrm{D}\left(\left|A_{0} / M_{3}\right| \gtrsim 0.35\right.$ and $\left.\tan \beta \gtrsim 5\right)$, at $M_{\text {SUSY }}$ scale we have $\left|M_{H_{d}}^{2}\right| \ll\left|M_{H_{u}}^{2}\right|$ and thus the CP-odd Higgs mass can be approximately given as

$$
m_{A}=\sqrt{\frac{\tan ^{2} \beta+1}{\tan ^{2} \beta-1}\left(M_{H_{d}}^{2}-M_{H_{u}}^{2}\right)-m_{Z}^{2}} \approx\left|M_{H_{u}}\right| \approx \mu .
$$

While like the point shown in eqs. (A.30), (A.31) in appendix A, for the samples in Class A and $\mathrm{B}\left(\left|A_{0} / M_{3}\right| \lesssim 0.35\right.$ and $\left.\tan \beta \gtrsim 35\right)$, at $M_{\text {SUSY }}$ scale $M_{H_{d}}^{2}$ can be comparable with $M_{H_{u}}^{2}$ and thus the CP-odd Higgs mass can be much smaller than the parameter $\mu$, especially for Class B where $M_{H_{d}}^{2}$ can be quite close to $M_{H_{u}}^{2}$. We can see these characteristics jointly from the right plane of figure 1, the left and middle planes of figure 2. In SUSY models, we have the following equation for $B_{s} \rightarrow \mu^{+} \mu^{-}$branch ratio

$$
\operatorname{Br}\left(B_{s} \rightarrow \mu^{+} \mu^{-}\right) \propto \frac{m_{t}^{4} \mu^{2} A_{t}^{2} \tan ^{6} \beta}{m_{A}^{4} m_{\tilde{t}}^{4}} .
$$

However, combing the three planes in figure 2 we notice that the large-tan $\beta(\gtrsim 30)$ samples with positive $M_{3}$ and small- $m_{A}(2-3 \mathrm{TeV})$ predict large $B_{s} \rightarrow \mu^{+} \mu^{-}$ratios, while the moderate-tan $\beta(35 \sim 40)$ samples with a negative $M_{3}$ and a small $m_{A}(2-3 \mathrm{TeV})$ predict small ratios. These positive- and negative- $M_{3}$ samples have different behaviors on $B_{s} \rightarrow$ $\mu^{+} \mu^{-}$, which is another new finding in our work.

In figure 3 , we project surviving samples in the $A_{0}$ versus $M_{0}$ (left), and $\tan \beta$ versus the lighter smuon mass $m_{\tilde{\mu}_{1}}$ (middle and right) planes, with colors indicating $m_{\tilde{\mu}_{1}}$ (left), SUSY contributions to muon g-2 $\Delta a_{\mu}$ (middle), and the lighter stau mass $m_{\tilde{\tau}_{1}}$ (right), respectively. In these three 2 -dimension planes, larger- $\Delta a_{\mu}$ samples are also shown on top of smaller ones. From the middle plane in figure 3, we can see that the muon g-2 anomaly can be solved in our scenario. In fact, light smuon and large $\tan \beta$ can give a sizable contribution to $\Delta a_{\mu}$ with positive $\mu$ in MSSM. Combined with figure 2 , we can see that the moderate-tan $\beta(35 \sim 40)$ samples with negative- $M_{3}$ and predicting small $\operatorname{Br}\left(B_{s} \rightarrow \mu^{+} \mu^{-}\right)$can contribute sizably to $\Delta a_{\mu}$ for light $\tilde{\mu}_{1}(250 \sim 450 \mathrm{GeV})$, but with heavy $\tilde{\tau}_{1}(\gtrsim 1 \mathrm{TeV})$ because of the exotic tuning among GUT parameters. This is also a new finding in this work. From the right plane we can know the light- $\tilde{\mu}_{1}$ and moderate$\tan \beta(10 \sim 45)$ regions with positive $M_{3}$ are missed mainly because of the lower bounds of stau mass $m_{\tilde{\tau}_{1}}$. The confusing missed part was shown in a figure in refs. $[27,55]$ but without an explanation, while we give a clear interpretation here in this work. 

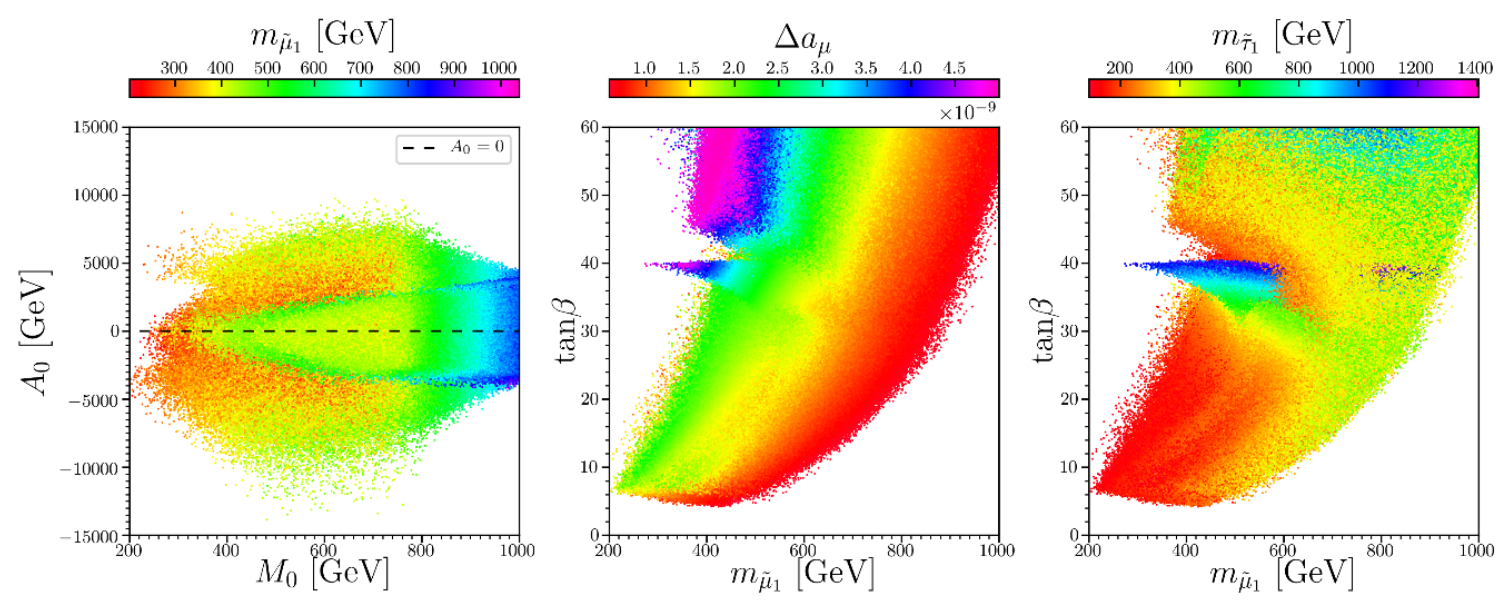

Figure 3. Surviving samples in the $A_{0}$ versus $M_{0}$ (left), and $\tan \beta$ versus the lighter smuon mass $m_{\tilde{\mu}_{1}}$ (middle and right) planes, with colors indicating $m_{\tilde{\mu}_{1}}$ (left), SUSY contributions to muon g-2 $\Delta a_{\mu}$ (middle), and the lighter stau mass $m_{\tilde{\tau}_{1}}$ (right), respectively. In these 2-dimension planes, larger- $\Delta a_{\mu}$ samples are projected on top of smaller ones.

When $\left|M_{3}\right| \gg\left|M_{1}\right|,\left|M_{2}\right|\left(M_{1,2,3}\right.$ are defined at GUT scale, and $M_{1,2,3}^{\text {SUSY }}$ hereafter are defined at $M_{\text {SUSY }}$ scale), the RGE running of $M_{3}$ can have a visible influence on $M_{1}$ and $M_{2}$. We checked in our samples that

$$
\begin{aligned}
\text { when } M_{3}>0,-200 & \lesssim M_{2}^{\text {SUSY }}-0.85 M_{2} \\
-80 & \lesssim 0 \mathrm{GeV}, \\
\text { when } M_{3}<0, \quad 0 & \lesssim M_{1}^{\text {SUSY }}-0.45 M_{1} \\
0 & \lesssim 0 \mathrm{GeV} ; \\
0 & \lesssim M_{1}^{\text {SUSY }}-0.45 M_{1} \lesssim 80 \mathrm{GeV},
\end{aligned}
$$

which can also be interpreted with the equations in appendix A. Since $M_{1}^{\mathrm{SUSY}}$ and $M_{2}^{\mathrm{SUSY}}$ are both in the diagonal position of the neutralino mass matrix, and $\mu \gg\left|M_{1}^{\mathrm{SUSY}}\right|,\left|M_{2}^{\mathrm{SUSY}}\right|$, the lightest neutralino (LSP) are either bino or wino, with nearly no mixing between them. The above discussions can be shown on the top planes in figure 4 . In all these 2-dimension planes in figure 4, larger relic density samples are also shown on top of smaller ones. From the bottom left plane, we can see that only bino-like LSP can generate enough relic density. While the wino neutralino always coannihilates with the wino charginos, the relic density is always too small to account for full abundance. We checked that for bino LSP, all the decay modes of wino neutralino $\chi_{2}^{0}$ and chargino $\chi_{1}^{ \pm}$contain a $\tau$ or $\tilde{\tau}_{1}$ final state. Thus, from the bottom right plane, we can see the searches for EW gauginos at the LHC set important constraints to the model. From the approximate equations and figure 4, we found a correlation between $M_{1,2}^{\text {SUSY }}$ and $M_{3}$, and its influence on dark matter composition, especially the deviation of boundaries from $\left|M_{1} / M_{2}\right|=2$ at the GUT scale. 

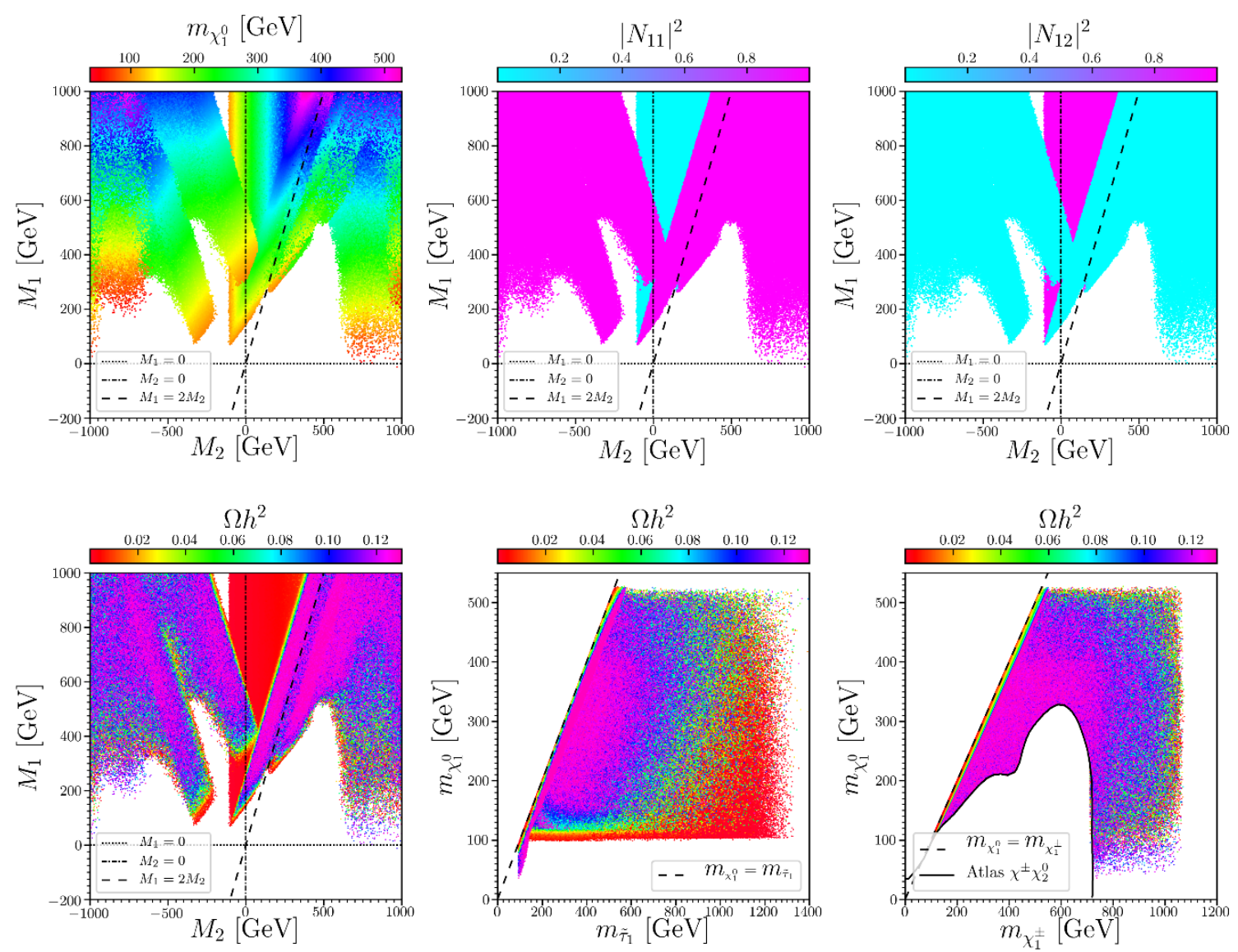

Figure 4. Surviving samples in the $M_{1}$ versus $M_{2}$ (upper 3 and lower left, where $M_{1,2}$ are both defined at GUT scale), LSP dark matter mass $m_{\chi_{1}^{0}}$ versus the lighter stau mass $m_{\tilde{\tau}_{1}}$ (lower middle), and $m_{\chi_{1}^{0}}$ versus the lighter chargino mass $m_{\chi_{1}^{ \pm}}$(lower right) planes. Colors indicate $m_{\chi_{1}^{0}}$ (upper left), bino component in LSP (upper middle), wino component (upper right), and LSP relic density $\left(\Omega h^{2}\right)$, respectively. In all these six 2-dimension planes, larger- $\Omega h^{2}$ samples are projected on top of smaller ones. In the lower right planes, the black solid curve indicate the constraint of searching for $\chi^{ \pm} \chi_{2}^{0}$ associate production in final states with tau leptons by Atlas collaboration at the $13 \mathrm{TeV}$ LHC [128].

From the bottom middle and right planes, we can also glimpse the annihilation mechanisms of bino-like LSP in our model. We checked that for samples predicting the right relic density $\left(0.107<\Omega h^{2}<0.131\right)$, there are mainly five single mechanisms ${ }^{5}$ and several combined ones: stau exchange $\left(\chi_{1}^{0} \chi_{1}^{0} \rightarrow \tau^{+} \tau^{-}\right)$, stau coannihilation $\left(\chi_{1}^{0} \tilde{\tau}_{1}^{ \pm} \rightarrow \tau^{ \pm} h / Z, W^{ \pm} \nu_{\tau}\right)$, chargino coannihilation $\left(\chi_{1}^{0} \chi_{1}^{ \pm} \rightarrow \ell^{ \pm} \nu_{\ell}\right)$, stau annihilation $\left(\tilde{\tau}_{1}^{+} \tilde{\tau}_{1}^{-} \rightarrow h h\right)$, other slepton annihilation $\left(\tilde{\ell} / \tilde{\nu}_{\ell} \tilde{\ell} / \tilde{\nu}_{\ell} \rightarrow X Y\right)$. Thus we sort our surviving samples into six classes by

\footnotetext{
${ }^{5}$ Notice that unlike that in CMSSM, we do not have surviving samples with stop as the next-to-lightest SUSY particle (NLSP), and stop coannihilation mechanism in our NUGM extension, which is because in our scenario we can have lighter bino-like neutralino and wino-like charginos for NUGM, and lighter sleptons to solve the muon g-2.
} 


\begin{tabular}{|c|c|c|c|}
\hline $\mathrm{P} 1, \tilde{\tau}_{1}$ coann. & $\mathrm{P} 2, \chi_{1}^{+}$coann. & P3, hybrid2 & $\mathrm{P} 7, \ell$ ann. \\
\hline$\overline{\chi_{1}^{0} \tilde{\tau}_{1} \rightarrow \tau h, 93.6 \%}$ & $\overline{\chi_{1}^{+} \chi_{2}^{0} \rightarrow u \bar{d}, 11.9 \%}$ & $\overline{\tilde{\tau}_{1} \tilde{\tau}_{1} \rightarrow h h, 18.4 \%}$ & $\overline{\tilde{\mu} \tilde{\nu}_{e}} \rightarrow e \nu_{\mu}, 11.3 \%$ \\
\hline$\chi_{1}^{0} \chi_{1}^{0} \rightarrow \tau \tau, 3.2 \%$ & $\chi_{1}^{+} \chi_{2}^{0} \rightarrow c \bar{s}, 11.9 \%$ & $\chi_{1}^{0} \tilde{\tau}_{1} \rightarrow \tau h, 9.7 \%$ & $\tilde{e} \tilde{\nu}_{\mu} \rightarrow \nu_{e} \mu, 11.3 \%$ \\
\hline$\chi_{1}^{0} \tilde{\tau}_{1} \rightarrow W^{-} \nu_{\tau}, 1.6 \%$ & $\chi_{1}^{+} \chi_{2}^{0} \rightarrow t \bar{b}, 11.4 \%$ & $\chi_{1}^{+} \chi_{2}^{0} \rightarrow u \bar{d}, 5.7 \%$ & $\tilde{\mu} \tilde{\nu}_{e} \rightarrow \nu_{e} \mu, 4.7 \%$ \\
\hline$\chi_{1}^{0} \tilde{\tau}_{1} \rightarrow Z \tau, 1.2 \%$ & $\chi_{1}^{+} \chi_{1}^{+} \rightarrow W^{+} W^{+}, 8.7 \%$ & $\chi_{1}^{+} \chi_{2}^{0} \rightarrow c \bar{s}, 5.7 \%$ & $\tilde{e} \tilde{\nu}_{\mu} \rightarrow e \nu_{\mu}, 4.7 \%$ \\
\hline & $\chi_{2}^{0} \chi_{2}^{0} \rightarrow W^{+} W^{-}, 8.7 \%$ & $\chi_{1}^{+} \chi_{2}^{0} \rightarrow t \bar{b}, 5.4 \%$ & $\tilde{\nu}_{e} \tilde{\bar{\nu}}_{e} \rightarrow W^{+} W^{-}, 4.6 \%$ \\
\hline P4, $\tilde{\tau}_{1}$ exch. & $Z W^{+}, 8.0 \%$ & $\chi_{2}^{0} \chi_{2}^{0} \rightarrow W^{+} W^{-}, 4.1 \%$ & $\tilde{\nu}_{\mu} \tilde{\bar{\nu}}_{\mu} \rightarrow W^{+} W^{-}, 4.6 \%$ \\
\hline$\chi_{1}^{0} \chi_{1}^{0} \rightarrow \tau$ & $Z Z, 5.2 \%$ & $\chi_{1}^{+} \chi_{1}^{+} \rightarrow W^{+} W^{+}, 4.1 \%$ & $\tilde{\nu}_{e} \tilde{\bar{\nu}}_{e} \rightarrow Z Z, 4.0 \%$ \\
\hline & ${ }_{1}^{+} \chi_{1}^{-} \rightarrow W^{+} W^{-}, 5.0 \%$ & $\chi_{1}^{+} \chi_{2}^{0} \rightarrow Z W^{+}, 3.8 \%$ & $\tilde{\nu}_{\mu} \tilde{\bar{\nu}}_{\mu} \rightarrow Z Z, 4.0 \%$ \\
\hline $\mathrm{P} 5, \tilde{\tau}$ & $x^{-} \rightarrow \gamma Z 30 \%$ & $\tilde{\tau}_{1} \tilde{\tau}_{1} \rightarrow Z Z, 2.5 \%$ & $\tilde{e} \tilde{\bar{\nu}} \tilde{\bar{\nu}}_{e} \rightarrow \gamma W^{-}, 2.4 \%$ \\
\hline $89.9 \%$ & $.0 \%$ & $\chi_{1}^{+} \chi_{1}^{-} \rightarrow Z Z, 2.5 \%$ & $\tilde{\mu} \tilde{\bar{\nu}}_{\mu} \rightarrow \gamma W^{-}, 2.4 \%$ \\
\hline$\chi_{1}^{0} \chi_{1}^{0} \rightarrow \tau \tau, 8.1 \%$ & $\chi_{1}^{-} \rightarrow s \bar{s}, 3.0 \%$ & $\chi_{1}^{+} \chi_{-}^{-} \rightarrow W^{+} W^{-} 2.4 \%$ & $\tilde{\nu}_{e} \tilde{\nu}_{\mu} \rightarrow \nu_{e} \nu_{\mu}, 2.3 \%$ \\
\hline & $\chi_{1}^{+} \chi_{1}^{-} \rightarrow d \bar{d}, 3.0 \%$ & $\chi_{1}^{+} \tilde{\tau}_{1} \rightarrow \nu_{\tau} h, 2.1 \%$ & $\tilde{\nu}_{e} \tilde{\nu}_{e} \rightarrow \nu_{e} \nu_{e}, 2.3 \%$ \\
\hline P6, $\tilde{\tau}_{1}$ hybrid3 & $\chi_{1}^{+} \chi_{1}^{-} \rightarrow b \bar{b}, 3.0 \%$ & $x_{1}+1>\tau \tau, 2.1 \% 0$ & $\tilde{\nu}_{\mu} \tilde{\nu}_{\mu} \rightarrow \nu_{\mu} \nu_{\mu}, 2.3 \%$ \\
\hline$\chi_{1}^{0} \tilde{\tau}_{1} \rightarrow \tau h, 89.7 \%$ & $\chi_{1}^{+} \chi_{1}^{-} \rightarrow u \bar{u}, 3.0 \%$ & & $\tilde{e} \tilde{\bar{\nu}}_{e} \rightarrow Z W^{-}, 2.0 \%$ \\
\hline$\chi_{1}^{0} \chi_{1}^{0} \rightarrow \tau \tau, 3.3 \%$ & $\chi_{1}^{+} \chi_{1}^{-} \rightarrow c \bar{c}, 3.0 \%$ & $\chi_{1}^{+} \chi_{1}^{0} \rightarrow \nu_{e} e^{+}, 1.9 \%$ & $\tilde{\mu} \tilde{\bar{\nu}}_{\mu} \rightarrow Z W^{-}, 2.0 \%$ \\
\hline$\chi_{1}^{0} \tilde{\tau}_{1} \rightarrow W^{-} \nu_{\tau}, 3.1$ & $\chi_{1}^{+} \chi_{1}^{-} \rightarrow t \bar{t}, 2.7 \%$ & $\chi_{1}^{+} \chi_{1}^{0} \rightarrow \nu_{\mu} \mu^{+}, 1.9 \%$ & $\tilde{e} \tilde{e} \rightarrow W^{+} W^{-}, 2.0 \%$ \\
\hline$\chi_{1}^{0} \tilde{\tau}_{1} \rightarrow Z \tau, 2.8 \%$ & $\chi_{1}^{+} \chi_{2}^{0} \rightarrow \gamma W^{+}, 2.0 \%$ & $\begin{array}{l}\tilde{\tau}_{1} \tilde{\tau}_{1} \rightarrow W^{+} W^{-}, 1.8 \% \\
\chi_{1}^{0} \tilde{\tau}_{1} \rightarrow Z \tau, 1.7 \%\end{array}$ & $\tilde{\mu} \tilde{\mu} \rightarrow W^{+} W^{-}, 2.0 \%$ \\
\hline
\end{tabular}

Table 2. The main annihilation channels and their relative contributions to $\langle\sigma v\rangle$ for the 7 benchmark points.

judging if it is a single or a combined mechanism:

$$
\begin{aligned}
& \tilde{\tau}_{1} \text { exchange }: m_{\tilde{\tau}_{1}}<200 \mathrm{GeV} \text {, } \\
& \tilde{\tau}_{1} \text { coannihilation : } \frac{m_{\tilde{\tau}_{1}}}{m_{\chi_{1}^{0}}}<1.2, \frac{m_{\chi_{1}^{ \pm}}}{m_{\chi_{1}^{0}}}>1.2, \\
& \chi_{1}^{ \pm} \text {coannihilation : } \frac{m_{\tilde{\tau}_{1}}}{m_{\chi_{1}^{0}}}>1.2, \frac{m_{\chi_{1}^{ \pm}}}{m_{\chi_{1}^{0}}}<1.2, \\
& \text { hybrid2 : } \frac{m_{\tilde{\tau}_{1}}}{m_{\chi_{1}^{0}}}<1.2, \frac{m_{\chi_{1}^{ \pm}}}{m_{\chi_{1}^{0}}}<1.2, \\
& \tilde{\tau}_{1} \text { hybrid3 }: \frac{m_{\tilde{\tau}_{1}}}{m_{\chi_{1}^{0}}}>1.2, \frac{m_{\chi_{1}^{ \pm}}}{m_{\chi_{1}^{0}}}>1.2,200<m_{\tilde{\tau}_{1}}<400 \mathrm{GeV}, \\
& \tilde{\ell} \text { annihilation : } \frac{m_{\tilde{\tau}_{1}}}{m_{\chi_{1}^{0}}}>1.2, \frac{m_{\chi_{1}^{ \pm}}}{m_{\chi_{1}^{0}}}>1.2, m_{\tilde{\tau}_{1}}>400 \mathrm{GeV} .
\end{aligned}
$$

For the hybrid2 samples, the dominated mechanism is a combined one by $\tilde{\tau}_{1}$ coannihilation and $\chi_{1}^{ \pm}$coannihilation; while for stau hybrid3, it is combined by $\tilde{\tau}_{1}$ exchange, $\tilde{\tau}_{1}$ coannihilation and $\tilde{\tau}_{1}$ annihilation, and the heavier $\tilde{\tau}_{1}$, the more annihilation and the less exchange; but when $\tilde{\tau}_{1}$ are heavier than $400 \mathrm{GeV}$, the dominated mechanism becomes other sleptons coannihilation, which is very complex in income and outcome particles. In table 2 , we give the detail annihilation information for 7 benchmark points. For each point, we list its main 


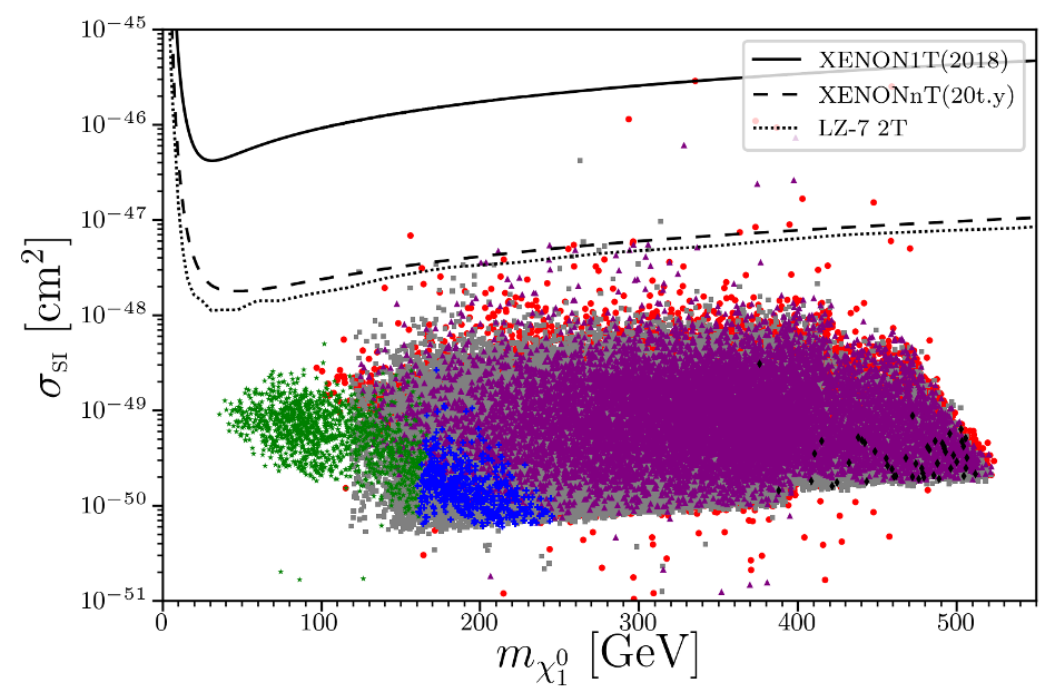

Figure 5. Surviving samples with sufficient dark matter relic density $\left(0.107<\Omega h^{2}<0.131\right)$ in the spin-independent DM-nucleon cross section $\sigma_{\mathrm{SI}}$ (original values without being rescaled by $\Omega / \Omega_{0}$ ) versus LSP DM mass $m_{\chi_{1}^{0}}$. The limits of XENON1T in 2018, the future detection sensitivity of XENONnT and LUX-ZEPLIN (LZ-7 2T) are shown by real, dashed and dotted curves respectively. Different annihilation scenarios of samples are also shown by different symbols with different colors: $\tilde{\tau}_{1}$ exchange by green star ' $\star$ ', $\tilde{\tau}_{1}$ hybrid3 by blue cross ' + ', $\tilde{\tau}_{1}$ coannihilation by red bullet ' $\bullet$ ', $\chi^{ \pm}$coannihilation by purple triangle ' $\mathbf{\Lambda}$ ', hybrid2 by gray square ' $\square$ ', and $\tilde{\ell}$ annihilation by black lozenge

annihilation channels and the relative contributions $(>1.5 \%)$ to $\langle\sigma v\rangle$. For completeness, we list the other information for the benchmark points in table 3 in appendix B. In this work, we show in detail the various annihilation mechanisms of DM, which is not done in refs. [27, 55]. And our findings is some different from these in refs. [8, 149] for NUGM version of pMSSM. We do not have annihilation mechanisms of $\mathrm{A} / \mathrm{H}$ funnel, focus point, and stop coannihilation, since our $H / A, \mu, \tilde{t}_{1}$ are much heavier, but we have stau exchange which may be omitted in refs. [8, 149].

In figure 5, we show the six classes of samples with sufficient relic density $(0.107<$ $\Omega h^{2}<0.131$ ) on the plane of SI DM-nucleon cross section $\sigma_{\mathrm{SI}}$ (original values without being rescaled by $\Omega / \Omega_{0}$ ) versus LSP mass $m_{\chi_{1}^{0}}$. We can see that, most of the samples predict small $\sigma_{\mathrm{SI}}$, which are over one order of magnitude lower than the future detection accuracy of $\mathrm{LZ}$ and XENONnT experiments. The $\sigma_{\mathrm{SI}}$ are smaller than these in refs. [27, 55], because we required sufficient relic density for these samples, and we checked that $\sigma_{\mathrm{SI}}$ can be larger for samples with insufficient relic density. However, a few samples corresponding to $\tilde{\tau}_{1}$ coannihilation, $\chi_{1}^{ \pm}$coannihilation and hybrid2 can be covered by the two detectors, with the LSP mass at about $200-400 \mathrm{GeV}$. It is because these samples have large percentages of coannihilation channels contributing to the DM relic density, which is also a new finding in this work. 


\section{Summary and conclusions}

We propose to generate non-universal gaugino masses in SU(5) GUT with the generalized Planck-scale mediation SUSY breaking mechanism, in which the non-universality arises from proper wavefunction normalization with lowest component VEVs of various high dimensional representations of the Higgs fields of SU(5) and an unique F-term VEV by the singlet. Different predictions on gaugino mass ratios with respect to widely studied scenarios are given. The gluino-SUGRA-like scenarios, where gluinos are much heavier than winos, bino and universal scalar masses, can be easily realized with appropriate combinations of such high-representation Higgs fields. With six GUT-scale free parameters in our scenario, we can solve elegantly the tension in mSUGRA between the muon g-2 and other constraints including the dark matter relic density and the direct sparticle search bounds from the LHC.

Taking into account the current constraints, we performed a scan and obtained the following observations:

- The large-tan $\beta(\gtrsim 35)$ samples with a moderate $M_{3}(\sim 5 \mathrm{TeV})$, a small $\left|A_{0} / M_{3}\right|$ $(\lesssim 0.4)$ and a small $m_{A}(\lesssim 4 \mathrm{TeV})$ are favoured to generate a $125 \mathrm{GeV}$ SM-like Higgs and predict a large muon g-2, while the stops mass and $\mu$ parameter, which are mainly determined by $\left|M_{3}\right|\left(\gg M_{0},\left|M_{1}\right|,\left|M_{2}\right|\right)$, can be about $6 \mathrm{TeV}$.

- The moderate-tan $\beta(35 \sim 40)$ samples with a negative $M_{3}$ can have a light smuon $(250 \sim 450 \mathrm{GeV})$ but a heavy stau ( $\gtrsim 1 \mathrm{TeV})$, which predict a large muon g-2 but small $\operatorname{Br}\left(B_{s} \rightarrow \mu^{+} \mu^{-}\right)$.

- The lightest neutralino can be as light as $100 \mathrm{GeV}$, which can predict a right relic abundance if it is bino-like and a much smaller relic density if it is wino-like.

- To obtain the right DM relic density, the annihilation mechanisms should be stau exchange, stau coannihilation, chargino coannihilation, slepton annihilation and the combination of two or three of them;

- To obtain the right DM relic density, the spin-independent DM-nucleon cross section is typically much smaller than the present bounds of XENON1T 2018, and an order of magnitude lower than the future detection sensitivity of LZ and XENONnT experiments.

\section{Acknowledgments}

This work was supported in part by the National Natural Science Foundation of China (NNSFC) under grant Nos. 11605123, 11675147, 11547103, 11547310, 11675242, 11851303, 11821505, by the Innovation Talent Project of Henan Province under grant number 15HASTIT017, by the Young Core Instructor Foundation of Henan Education Department, by Peng-Huan-Wu Theoretical Physics Innovation Center (11747601), by the CAS Center for Excellence in Particle Physics (CCEPP), by the CAS Key Research Program 
of Frontier Sciences and by a Key R\&D Program of Ministry of Science and Technology under number 2017YFA0402200-04. JZ also thanks Prof. Stephen P. Martin for helpful discussion, thanks the support of the China Scholarship Council (CSC) under Grant No.201706275160 while he was working at the University of Chicago as a visiting scholar, and thanks the support of the US National Science Foundation (NSF) under Grant No. PHY-0855561 while he was working at Michigan State University.

\section{A Correlations between the parameters at SUSY scale and GUT scale}

We show the correlations between the parameters at soft SUSY scale and GUT scale. For the benchmark point P4, the GUT scale is calculated to be $M_{\mathrm{GUT}}=1.27 \times 10^{16} \mathrm{GeV}$. Then we use two-loop RGEs to run the parameters from GUT scale to the SUSY scale, which we choose as $M_{\mathrm{SUSY}}=4 \mathrm{TeV}$. We repeat this process over 15 times by slightly changing the following one or two parameters excluding $\tan \beta$ at GUT scale each time

$$
p_{j, k}^{\mathrm{GUT}}=A_{0}, M_{0}, M_{1}, M_{2}, M_{3} .
$$

For the linear-correlation parameters

$$
p_{i}^{\mathrm{SUSY}}=A_{t}, A_{\tau}, A_{\mu}, M_{1}^{\mathrm{SUSY}}, M_{2}^{\mathrm{SUSY}}, M_{3}^{\mathrm{SUSY}}
$$

we calculate the coefficients by

$$
C_{i j}=\frac{\Delta p_{i}^{\mathrm{SUSY}}}{\Delta p_{j}^{\mathrm{GUT}}} .
$$

For the quadratic-correlation parameters

$$
p_{i}^{\mathrm{SUSY}}=\mu^{2}, M_{H_{u}}^{2}, M_{H_{d}}^{2}, M_{Q_{3}}^{2}, M_{U_{3}}^{2}, M_{L_{3}}^{2}, M_{E_{3}}^{2}, M_{L_{2}}^{2}, M_{E_{2}}^{2}
$$

we calculate the coefficients by

$$
C_{i j k(k \geqslant j)}=\frac{n \Delta p_{i}^{\mathrm{SUSY}}}{\Delta p_{j}^{\mathrm{GUT}} \Delta p_{k}^{\mathrm{GUT}}} \quad(n=2 \text { for } k=j, n=1 \text { for } k>j),
$$

which can be written in a $5 \times 5$ triangular-matrix for each parameter. In the following equations, we list the coefficients for the benchmark point P4 in Class $\mathrm{C}$ and D in eq. (4.1), and P7 in Class A and B in eq. (4.1). We checked that these coefficients coincide approximately with our parameter-running results in NMSSMTools-5.2.0. Most of these equations (except $M_{H_{d}}^{2}$ and $m_{A}^{2}$, for example) can be generalized roughly to other surviving samples in their represented classes, because all of them satisfy $\tan \beta \gg 1$. However, most coefficients will change a lot if one change the SUSY scale too much, e.g., to $M_{\mathrm{SUSY}}=400 \mathrm{GeV}$ as in ref. [28]. These equations are given as follows.

For Benchmark Point P4 (with $\tan \beta=20.8$ fixed and on behalf of Class C and D),

$$
\begin{aligned}
& A_{t}=0.40 A_{0}-1.16 M_{3}-0.04 M_{0}-0.04 M_{1}-0.18 M_{2} \\
& A_{\tau}=0.93 A_{0}-0.40 M_{2}-0.14 M_{1}+0.028 M_{3}
\end{aligned}
$$




$$
\begin{aligned}
& A_{\mu}=0.96 A_{0}-0.41 M_{2}-0.14 M_{1}+0.028 M_{3} \\
& M_{1}^{\mathrm{SUSY}}=0.46 M_{1}-0.007 M_{3} \\
& M_{2}^{\mathrm{SUSY}}=0.83 M_{2}-0.018 M_{3} \\
& M_{3}^{\mathrm{SUSY}}=1.92 M_{3}-0.08 M_{2}+0.04 M_{0} \\
& M_{\tilde{Q}_{3}}^{2}=\left(\begin{array}{lllll}
A_{0} & M_{0} & M_{1} & M_{2} & M_{3}
\end{array}\right)\left(\begin{array}{ccccc}
-0.067 & -0.29 & -0.01 & 0.04 & 0.09 \\
\mathbf{0} & -1.01 & -0.30 & 0.43 & 0.26 \\
\mathbf{0} & \mathbf{0} & 0.11 & 0.01 & 0.01 \\
\mathbf{0} & \mathbf{0} & \mathbf{0} & 1.05 & -0.51 \\
\mathbf{0} & \mathbf{0} & \mathbf{0} & \mathbf{0} & 2.15
\end{array}\right)\left(\begin{array}{c}
A_{0} \\
M_{0} \\
M_{1} \\
M_{2} \\
M_{3}
\end{array}\right) \\
& M_{\tilde{U}_{3}}^{2}=\left(\begin{array}{lllll}
A_{0} & M_{0} & M_{1} & M_{2} & M_{3}
\end{array}\right)\left(\begin{array}{ccccc}
-0.096 & -0.24 & -0.00 & 0.05 & 0.15 \\
\mathbf{0} & -0.99 & -0.25 & 0.35 & 0.22 \\
\mathbf{0} & \mathbf{0} & 0.13 & 0.01 & 0.00 \\
\mathbf{0} & \mathbf{0} & \mathbf{0} & 0.51 & -0.44 \\
\mathbf{0} & \mathbf{0} & \mathbf{0} & \mathbf{0} & 1.92
\end{array}\right)\left(\begin{array}{c}
A_{0} \\
M_{0} \\
M_{1} \\
M_{2} \\
M_{3}
\end{array}\right) \\
& M_{H_{u}}^{2}=\left(\begin{array}{lllll}
A_{0} & M_{0} & M_{1} & M_{2} & M_{3}
\end{array}\right)\left(\begin{array}{ccccc}
-0.102 & 0.20 & 0.02 & 0.03 & 0.22 \\
\mathbf{0} & 1.18 & 0.19 & -0.28 & -0.16 \\
\mathbf{0} & \mathbf{0} & -0.06 & -0.01 & -0.03 \\
\mathbf{0} & \mathbf{0} & \mathbf{0} & -0.23 & 0.18 \\
\mathbf{0} & \mathbf{0} & \mathbf{0} & \mathbf{0} & -0.91
\end{array}\right)\left(\begin{array}{c}
A_{0} \\
M_{0} \\
M_{1} \\
M_{2} \\
M_{3}
\end{array}\right) \\
& M_{H_{d}}^{2}=\left(\begin{array}{lllll}
A_{0} & M_{0} & M_{1} & M_{2} & M_{3}
\end{array}\right)\left(\begin{array}{ccccc}
-0.027 & 0.03 & 0.00 & 0.01 & 0.04 \\
\mathbf{0} & 0.99 & 0.02 & -0.03 & -0.02 \\
\mathbf{0} & \mathbf{0} & 0.02 & -0.00 & -0.00 \\
\mathbf{0} & \mathbf{0} & \mathbf{0} & 0.31 & 0.00 \\
\mathbf{0} & \mathbf{0} & \mathbf{0} & \mathbf{0} & -0.09
\end{array}\right)\left(\begin{array}{c}
A_{0} \\
M_{0} \\
M_{1} \\
M_{2} \\
M_{3}
\end{array}\right) \\
& \mu^{2}=\frac{M_{H_{d}}^{2}-M_{H_{u}}^{2} \tan ^{2} \beta}{\tan ^{2} \beta-1}-\frac{m_{Z}^{2}}{2} \\
& \approx\left(\begin{array}{lllll}
A_{0} & M_{0} & M_{1} & M_{2} & M_{3}
\end{array}\right)\left(\begin{array}{ccccc}
0.102 & -0.20 & -0.02 & -0.03 & -0.22 \\
\mathbf{0} & -1.18 & -0.19 & 0.28 & 0.16 \\
\mathbf{0} & \mathbf{0} & 0.06 & 0.01 & 0.03 \\
\mathbf{0} & \mathbf{0} & \mathbf{0} & 0.24 & -0.18 \\
\mathbf{0} & \mathbf{0} & \mathbf{0} & \mathbf{0} & 0.91
\end{array}\right)\left(\begin{array}{l}
A_{0} \\
M_{0} \\
M_{1} \\
M_{2} \\
M_{3}
\end{array}\right) \\
& m_{A}^{2}=\frac{\tan ^{2} \beta+1}{\tan ^{2} \beta-1}\left(M_{H_{d}}^{2}-M_{H_{u}}^{2}\right)-m_{Z}^{2} \\
& \approx\left(\begin{array}{lllll}
A_{0} & M_{0} & M_{1} & M_{2} & M_{3}
\end{array}\right)\left(\begin{array}{ccccc}
0.075 & -0.18 & -0.02 & -0.02 & -0.19 \\
\mathbf{0} & -0.19 & -0.17 & 0.25 & 0.15 \\
\mathbf{0} & \mathbf{0} & 0.09 & 0.01 & 0.02 \\
\mathbf{0} & \mathbf{0} & \mathbf{0} & 0.54 & -0.18 \\
\mathbf{0} & \mathbf{0} & \mathbf{0} & \mathbf{0} & 0.82
\end{array}\right)\left(\begin{array}{c}
A_{0} \\
M_{0} \\
M_{1} \\
M_{2} \\
M_{3}
\end{array}\right)
\end{aligned}
$$




$$
\begin{gathered}
M_{\tilde{L}_{3}}^{2}=\left(\begin{array}{lllll}
A_{0} & M_{0} & M_{1} & M_{2} & M_{3}
\end{array}\right)\left(\begin{array}{ccccc}
-0.011 & 0.00 & 0.00 & 0.00 & -0.00 \\
\mathbf{0} & 0.96 & 0.00 & 0.00 & -0.00 \\
\mathbf{0} & \mathbf{0} & 0.03 & -0.00 & -0.00 \\
\mathbf{0} & \mathbf{0} & \mathbf{0} & 0.37 & -0.00 \\
\mathbf{0} & \mathbf{0} & \mathbf{0} & \mathbf{0} & -0.004
\end{array}\right)\left(\begin{array}{l}
A_{0} \\
M_{0} \\
M_{1} \\
M_{2} \\
M_{3}
\end{array}\right) \\
M_{\tilde{L}_{2}}^{2}=\left(\begin{array}{lllll}
A_{0} & M_{0} & M_{1} & M_{2} & M_{3}
\end{array}\right)\left(\begin{array}{ccccc}
0.000 & 0.00 & -0.00 & -0.00 & 0.00 \\
\mathbf{0} & 0.99 & 0.00 & 0.00 & -0.00 \\
\mathbf{0} & \mathbf{0} & 0.03 & -0.00 & -0.00 \\
\mathbf{0} & \mathbf{0} & \mathbf{0} & 0.38 & -0.00 \\
\mathbf{0} & \mathbf{0} & \mathbf{0} & \mathbf{0} & -0.004
\end{array}\right)\left(\begin{array}{c}
A_{0} \\
M_{0} \\
M_{1} \\
M_{2} \\
M_{3}
\end{array}\right) \\
M_{\tilde{E}_{3}}^{2}=\left(\begin{array}{llllll}
A_{0} & M_{0} & M_{1} & M_{2} & M_{3}
\end{array}\right)\left(\begin{array}{ccccc}
-0.022 & 0.00 & 0.00 & 0.01 & -0.00 \\
\mathbf{0} & 0.93 & 0.00 & -0.00 & 0.00 \\
\mathbf{0} & \mathbf{0} & 0.13 & -0.00 & -0.00 \\
\mathbf{0} & \mathbf{0} & \mathbf{0} & -0.01 & 0.00 \\
\mathbf{0} & \mathbf{0} & \mathbf{0} & \mathbf{0} & -0.000
\end{array}\right)\left(\begin{array}{c}
A_{0} \\
M_{0} \\
M_{1} \\
M_{2} \\
M_{3}
\end{array}\right) \\
M_{\tilde{E}_{2}}^{2}=\left(\begin{array}{llllll}
A_{0} & M_{0} & M_{1} & M_{2} & M_{3}
\end{array}\right)\left(\begin{array}{ccccc}
0.000 & 0.00 & -0.00 & 0.00 & -0.00 \\
\mathbf{0} & 1.00 & 0.00 & -0.00 & -0.00 \\
\mathbf{0} & \mathbf{0} & 0.13 & -0.00 & -0.00 \\
\mathbf{0} & \mathbf{0} & \mathbf{0} & -0.00 & 0.00 \\
\mathbf{0} & \mathbf{0} & \mathbf{0} & \mathbf{0} & -0.001
\end{array}\right)\left(\begin{array}{l}
A_{0} \\
M_{0} \\
M_{1} \\
M_{2} \\
M_{3}
\end{array}\right)
\end{gathered}
$$

For benchmark point $\mathrm{P} 7$ in Class $\mathrm{A}$ and $\mathrm{B}$ (with $\tan \beta=39.7$ ), we have

$$
\begin{aligned}
A_{t} & =0.31 A_{0}-1.09 M_{3}-0.03 M_{1}-0.11 M_{2} \\
A_{\tau} & =0.50 A_{0}-0.45 M_{2}-0.12 M_{1}+0.242 M_{3} \\
A_{\mu} & =0.65 A_{0}-0.50 M_{2}-0.13 M_{1}+0.257 M_{3} \\
M_{1}^{\mathrm{SUSY}} & =0.46 M_{1}-0.007 M_{3} \\
M_{2}^{\mathrm{SUSY}} & =0.83 M_{2}-0.017 M_{3} \\
M_{3}^{\mathrm{SUSY}} & =1.93 M_{3}-0.17 M_{2} \\
M_{\tilde{Q}_{3}}^{2} & =\left(\begin{array}{llllll}
A_{0} & M_{0} M_{1} M_{2} M_{3}
\end{array}\right)\left(\begin{array}{ccccc}
-0.07 & 0.02 & 0.01 & -0.01 & 0.13 \\
\mathbf{0} & 3.09 & -0.21 & -3.86 & 0.59 \\
\mathbf{0} & \mathbf{0} & 0.03 & -0.16 & -0.02 \\
\mathbf{0} & \mathbf{0} & \mathbf{0} & 4.06 & -1.21 \\
\mathbf{0} & \mathbf{0} & \mathbf{0} & \mathbf{0} & 1.96
\end{array}\right)\left(\begin{array}{c}
A_{0} \\
M_{0} \\
M_{1} \\
M_{2} \\
M_{3}
\end{array}\right) \\
M_{\tilde{U}_{3}}^{2} & =\left(\begin{array}{lllll}
A_{0} M_{0} M_{1} M_{2} M_{3}
\end{array}\right)\left(\begin{array}{ccccc}
-0.06 & 0.03 & 0.01 & -0.00 & 0.11 \\
\mathbf{0} & 3.12 & -0.21 & -4.00 & 0.62 \\
\mathbf{0} & \mathbf{0} & 0.08 & -0.16 & -0.02 \\
\mathbf{0} & \mathbf{0} & \mathbf{0} & 3.81 & -1.30 \\
\mathbf{0} & \mathbf{0} & \mathbf{0} & \mathbf{0} & 1.96
\end{array}\right)\left(\begin{array}{l}
A_{0} \\
M_{0} \\
M_{1} \\
M_{2} \\
M_{3}
\end{array}\right)
\end{aligned}
$$




$$
\begin{aligned}
& M_{H_{u}}^{2}=\left(\begin{array}{lllll}
A_{0} & M_{0} & M_{1} & M_{2} & M_{3}
\end{array}\right)\left(\begin{array}{ccccc}
-0.09 & -0.01 & 0.01 & 0.07 & 0.18 \\
\mathbf{0} & -1.57 & 0.17 & 2.48 & -0.37 \\
\mathbf{0} & \mathbf{0} & -0.02 & 0.10 & -0.01 \\
\mathbf{0} & \mathbf{0} & \mathbf{0} & -2.30 & 0.71 \\
\mathbf{0} & \mathbf{0} & \mathbf{0} & \mathbf{0} & -0.899
\end{array}\right)\left(\begin{array}{c}
A_{0} \\
M_{0} \\
M_{1} \\
M_{2} \\
M_{3}
\end{array}\right) \\
& M_{H_{d}}^{2}=\left(\begin{array}{lllll}
A_{0} & M_{0} & M_{1} & M_{2} & M_{3}
\end{array}\right)\left(\begin{array}{ccccc}
-0.15 & -0.02 & 0.01 & 0.08 & 0.24 \\
\mathbf{0} & -1.46 & 0.19 & 2.50 & -0.38 \\
\mathbf{0} & \mathbf{0} & -0.01 & 0.10 & 0.00 \\
\mathbf{0} & \mathbf{0} & \mathbf{0} & -2.45 & 0.96 \\
\mathbf{0} & \mathbf{0} & \mathbf{0} & \mathbf{0} & -0.810
\end{array}\right)\left(\begin{array}{c}
A_{0} \\
M_{0} \\
M_{1} \\
M_{2} \\
M_{3}
\end{array}\right) \\
& \mu^{2}=\frac{M_{H_{d}}^{2}-M_{H_{u}}^{2} \tan ^{2} \beta}{\tan ^{2} \beta-1}-\frac{m_{Z}^{2}}{2} \\
& \approx\left(\begin{array}{lllll}
A_{0} & M_{0} & M_{1} & M_{2} & M_{3}
\end{array}\right)\left(\begin{array}{ccccc}
0.09 & 0.01 & -0.01 & -0.07 & -0.18 \\
\mathbf{0} & 1.59 & -0.17 & -2.50 & 0.37 \\
\mathbf{0} & \mathbf{0} & 0.02 & -0.10 & 0.01 \\
\mathbf{0} & \mathbf{0} & \mathbf{0} & 2.30 & -0.70 \\
\mathbf{0} & \mathbf{0} & \mathbf{0} & \mathbf{0} & 0.899
\end{array}\right)\left(\begin{array}{l}
A_{0} \\
M_{0} \\
M_{1} \\
M_{2} \\
M_{3}
\end{array}\right) \\
& m_{A}^{2}=\frac{\tan ^{2} \beta+1}{\tan ^{2} \beta-1}\left(M_{H_{d}}^{2}-M_{H_{u}}^{2}\right)-m_{Z}^{2} \\
& \approx\left(\begin{array}{lllll}
A_{0} & M_{0} & M_{1} & M_{2} & M_{3}
\end{array}\right)\left(\begin{array}{cccccc}
-0.07 & -0.01 & 0.00 & 0.01 & 0.06 \\
\mathbf{0} & 0.11 & 0.02 & 0.01 & -0.00 \\
\mathbf{0} & \mathbf{0} & 0.01 & -0.00 & 0.01 \\
\mathbf{0} & \mathbf{0} & \mathbf{0} & -0.15 & 0.25 \\
\mathbf{0} & \mathbf{0} & \mathbf{0} & \mathbf{0} & 0.089
\end{array}\right)\left(\begin{array}{l}
A_{0} \\
M_{0} \\
M_{1} \\
M_{2} \\
M_{3}
\end{array}\right) \\
& M_{\tilde{L}_{3}}^{2}=\left(\begin{array}{lllll}
A_{0} & M_{0} & M_{1} & M_{2} & M_{3}
\end{array}\right)\left(\begin{array}{cccccc}
-0.02 & -0.00 & 0.01 & 0.02 & -0.01 \\
\mathbf{0} & 0.83 & -0.01 & -0.01 & -0.00 \\
\mathbf{0} & \mathbf{0} & 0.03 & -0.00 & 0.00 \\
\mathbf{0} & \mathbf{0} & \mathbf{0} & 0.39 & -0.02 \\
\mathbf{0} & \mathbf{0} & \mathbf{0} & \mathbf{0} & 0.009
\end{array}\right)\left(\begin{array}{c}
A_{0} \\
M_{0} \\
M_{1} \\
M_{2} \\
M_{3}
\end{array}\right) \\
& M_{\tilde{L}_{2}}^{2}=\left(\begin{array}{lllll}
A_{0} & M_{0} & M_{1} & M_{2} & M_{3}
\end{array}\right)\left(\begin{array}{ccccc}
0.00 & -0.00 & -0.00 & -0.00 & -0.00 \\
\mathbf{0} & 0.96 & -0.00 & 0.04 & -0.01 \\
\mathbf{0} & \mathbf{0} & 0.03 & 0.00 & -0.00 \\
\mathbf{0} & \mathbf{0} & \mathbf{0} & 0.35 & 0.00 \\
\mathbf{0} & \mathbf{0} & \mathbf{0} & \mathbf{0} & -0.005
\end{array}\right)\left(\begin{array}{c}
A_{0} \\
M_{0} \\
M_{1} \\
M_{2} \\
M_{3}
\end{array}\right) \\
& M_{\tilde{E}_{3}}^{2}=\left(\begin{array}{lllll}
A_{0} & M_{0} & M_{1} & M_{2} & M_{3}
\end{array}\right)\left(\begin{array}{ccccc}
-0.05 & -0.00 & 0.01 & 0.04 & -0.02 \\
\mathbf{0} & 0.74 & -0.01 & -0.10 & 0.01 \\
\mathbf{0} & \mathbf{0} & 0.12 & -0.01 & 0.00 \\
\mathbf{0} & \mathbf{0} & \mathbf{0} & 0.06 & -0.04 \\
\mathbf{0} & \mathbf{0} & \mathbf{0} & \mathbf{0} & 0.026
\end{array}\right)\left(\begin{array}{c}
A_{0} \\
M_{0} \\
M_{1} \\
M_{2} \\
M_{3}
\end{array}\right)
\end{aligned}
$$




$$
M_{\tilde{E}_{2}}^{2}=\left(\begin{array}{lllll}
A_{0} & M_{0} & M_{1} & M_{2} & M_{3}
\end{array}\right)\left(\begin{array}{cccccc}
-0.00 & -0.00 & -0.00 & -0.00 & -0.00 \\
\mathbf{0} & 0.99 & -0.00 & 0.01 & -0.00 \\
\mathbf{0} & \mathbf{0} & 0.13 & 0.00 & -0.00 \\
\mathbf{0} & \mathbf{0} & \mathbf{0} & -0.00 & 0.00 \\
\mathbf{0} & \mathbf{0} & \mathbf{0} & \mathbf{0} & -0.001
\end{array}\right)\left(\begin{array}{c}
A_{0} \\
M_{0} \\
M_{1} \\
M_{2} \\
M_{3}
\end{array}\right)
$$

\section{B The detail information of the 7 benchmark points}

As a supplement to table 2, we list in table 3 the model parameters, relevant sparticle masses and phenomenological observables for the 7 benchmark points.

\begin{tabular}{|c|c|c|c|c|c|c|c|}
\hline & $\mathrm{P} 1$ & $\mathrm{P} 2$ & P3 & $\mathrm{P} 4$ & P5 & $\mathrm{P} 6$ & P7 \\
\hline $\tan \beta$ & 54.1 & 28.5 & 29.1 & 20.8 & 58.3 & 48.5 & 39.7 \\
\hline$A_{0}[\mathrm{GeV}]$ & 2607 & 2410 & -72 & -2250 & 1516 & -1227 & 363 \\
\hline$M_{0}[\mathrm{GeV}]$ & 979 & 808 & 736 & 564 & 718 & 624 & 577 \\
\hline$M_{1}[\mathrm{GeV}]$ & 772 & 850 & 813 & 291 & 548 & 773 & 712 \\
\hline$M_{2}[\mathrm{GeV}]$ & -819 & 376 & -685 & 971 & -988 & 967 & -713 \\
\hline$M_{3}[\mathrm{GeV}]$ & 9881 & -6080 & -8350 & 6335 & 6032 & 6734 & -9137 \\
\hline$\mu[\mathrm{GeV}]$ & 9075 & 6338 & 8081 & 6619 & 5788 & 6808 & 8786 \\
\hline$m_{h}[\mathrm{GeV}]$ & 126.2 & 124.9 & 125.2 & 125.1 & 125.2 & 125.8 & 125.7 \\
\hline$m_{A}[\mathrm{GeV}]$ & 6861 & 5011 & 6570 & 6407 & 4276 & 5145 & 2596 \\
\hline$m_{\tilde{t}_{1}}[\mathrm{GeV}]$ & 13819 & 8634 & 11742 & 8870 & 8720 & 9491 & 12744 \\
\hline$m_{\tilde{\tau}_{1}}[\mathrm{GeV}]$ & 336 & 543 & 477 & 120 & 250 & 350 & 1015 \\
\hline$m_{\tilde{\mu}_{1}}[\mathrm{GeV}]$ & 912 & 754 & 627 & 540 & 707 & 641 & 388 \\
\hline$m_{\tilde{\nu}_{1}}[\mathrm{GeV}]$ & 926 & 751 & 625 & 672 & 874 & 718 & 390 \\
\hline$m_{\tilde{\chi}_{1}^{ \pm}}[\mathrm{GeV}]$ & 920 & 451 & 460 & 738 & 986 & 734 & 485 \\
\hline $\operatorname{Br}\left(B_{s} \rightarrow \mu^{+} \mu^{-}\right)\left[10^{-9}\right]$ & 3.67 & 3.53 & 3.55 & 3.58 & 3.89 & 3.75 & 3.20 \\
\hline$B r\left(B_{d} \rightarrow \mu^{+} \mu^{-}\right)\left[10^{-10}\right]$ & 1.05 & 1.00 & 1.00 & 1.01 & 1.14 & 1.06 & 0.87 \\
\hline$B r(b \rightarrow s \gamma)\left[10^{-4}\right]$ & 3.35 & 3.38 & 3.37 & 3.36 & 3.36 & 3.36 & 3.44 \\
\hline$\Delta a_{\mu}^{\text {SUSY }}\left[10^{-10}\right]$ & 7.81 & 6.51 & 10.3 & 6.36 & 7.95 & 18.0 & 46.0 \\
\hline$m_{\tilde{\chi}_{1}^{0}}[\mathrm{GeV}]$ & 284.8 & 423.8 & 426.9 & 86.9 & 205.2 & 303.8 & 386.0 \\
\hline$m_{\tilde{\chi}_{2}^{0}}[\mathrm{GeV}]$ & 920 & 451 & 460 & 738 & 986 & 734 & 485 \\
\hline$\sigma_{\mathrm{SI}}\left[10^{-50} \mathrm{~cm}^{-1}\right]$ & 0.91 & 6.63 & 3.04 & 3.58 & 3.16 & 2.68 & 2.23 \\
\hline$\sigma_{\mathrm{P}}^{\mathrm{SD}}\left[10^{-47} \mathrm{~cm}^{-1}\right]$ & 1.29 & 5.31 & 1.99 & 4.17 & 7.82 & 3.95 & 1.44 \\
\hline$\sigma_{\mathrm{N}}^{\mathrm{SD}}\left[10^{-47} \mathrm{~cm}^{-1}\right]$ & 0.97 & 3.47 & 1.40 & 2.62 & 5.81 & 2.65 & 1.01 \\
\hline$\Omega h^{2}$ & 0.109 & 0.112 & 0.120 & 0.111 & 0.130 & 0.126 & 0.118 \\
\hline
\end{tabular}

Table 3. The detail information of the 7 benchmark points. 
Open Access. This article is distributed under the terms of the Creative Commons Attribution License (CC-BY 4.0), which permits any use, distribution and reproduction in any medium, provided the original author(s) and source are credited.

\section{References}

[1] ATLAS collaboration, Observation of a new particle in the search for the Standard Model Higgs boson with the ATLAS detector at the LHC, Phys. Lett. B 716 (2012) 1 [arXiv:1207.7214] [INSPIRE].

[2] CMS collaboration, Observation of a new boson at a mass of $125 \mathrm{GeV}$ with the CMS experiment at the LHC, Phys. Lett. B 716 (2012) 30 [arXiv:1207.7235] [INSPIRE].

[3] Muon $g-2$ collaboration, G.W. Bennett et al., Final Report of the Muon E821 Anomalous Magnetic Moment Measurement at BNL, Phys. Rev. D 73 (2006) 072003 [hep-ex/0602035] [INSPIRE].

[4] J. Cao, Z. Heng, D. Li and J.M. Yang, Current experimental constraints on the lightest Higgs boson mass in the constrained MSSM, Phys. Lett. B 710 (2012) 665 [arXiv:1112.4391] [INSPIRE].

[5] J. Ellis and K.A. Olive, Revisiting the Higgs Mass and Dark Matter in the CMSSM, Eur. Phys. J. C 72 (2012) 2005 [arXiv:1202.3262] [InSPIRE].

[6] C. Han, K.-i. Hikasa, L. Wu, J.M. Yang and Y. Zhang, Status of CMSSM in light of current LHC Run-2 and LUX data, Phys. Lett. B 769 (2017) 470 [arXiv:1612.02296] [INSPIRE].

[7] P. Bechtle et al., Killing the CMSSM softly, Eur. Phys. J. C 76 (2016) 96 [arXiv: 1508.05951] [INSPIRE].

[8] E.A. Bagnaschi et al., Supersymmetric Dark Matter after LHC Run 1, Eur. Phys. J. C 75 (2015) 500 [arXiv: 1508.01173] [INSPIRE].

[9] GAMBIT collaboration, P. Athron et al., Global fits of GUT-scale SUSY models with GAMBIT, Eur. Phys. J. C 77 (2017) 824 [arXiv:1705.07935] [InSPIRE].

[10] M. Kubo, J. Sato, T. Shimomura, Y. Takanishi and M. Yamanaka, Big-bang nucleosynthesis and leptogenesis in the CMSSM, Phys. Rev. D 97 (2018) 115013 [arXiv:1803.07686] [INSPIRE].

[11] S. Banerjee, G. Bélanger, B. Mukhopadhyaya and P.D. Serpico, Signatures of sneutrino dark matter in an extension of the CMSSM, JHEP 07 (2016) 095 [arXiv:1603.08834] [INSPIRE].

[12] I. Gogoladze, B. He, A. Mustafayev, S. Raza and Q. Shafi, Effects of Neutrino Inverse Seesaw Mechanism on the Sparticle Spectrum in CMSSM and NUHM2, JHEP 05 (2014) 078 [arXiv: 1401.8251] [INSPIRE].

[13] J.F. Gunion, Y. Jiang and S. Kraml, The Constrained NMSSM and Higgs near $125 \mathrm{GeV}$, Phys. Lett. B 710 (2012) 454 [arXiv:1201.0982] [INSPIRE].

[14] U. Ellwanger and C. Hugonie, Higgs bosons near $125 \mathrm{GeV}$ in the NMSSM with constraints at the GUT scale, Adv. High Energy Phys. 2012 (2012) 625389 [arXiv:1203.5048] [INSPIRE].

[15] K. Kowalska, S. Munir, L. Roszkowski, E.M. Sessolo, S. Trojanowski and Y.-L.S. Tsai, Constrained next-to-minimal supersymmetric standard model with a $126 \mathrm{GeV}$ Higgs boson: A global analysis, Phys. Rev. D 87 (2013) 115010 [arXiv:1211.1693] [InSPIRE]. 
[16] C. Beskidt, W. de Boer and D.I. Kazakov, A comparison of the Higgs sectors of the CMSSM and NMSSM for a 126 GeV Higgs boson, Phys. Lett. B 726 (2013) 758 [arXiv:1308.1333] [INSPIRE].

[17] D. Kim, P. Athron, C. Balázs, B. Farmer and E. Hutchison, Bayesian naturalness of the CMSSM and CNMSSM, Phys. Rev. D 90 (2014) 055008 [arXiv:1312.4150] [INSPIRE].

[18] A. Fowlie, Is the CNMSSM more credible than the CMSSM?, Eur. Phys. J. C 74 (2014) 3105 [arXiv: 1407.7534] [INSPIRE].

[19] A. Choudhury, S. Rao and L. Roszkowski, Impact of LHC data on muon g- 2 solutions in a vectorlike extension of the constrained MSSM, Phys. Rev. D 96 (2017) 075046 [arXiv: 1708.05675] [INSPIRE].

[20] A. Choudhury, L. Darmé, L. Roszkowski, E.M. Sessolo and S. Trojanowski, Muon g-2 and related phenomenology in constrained vector-like extensions of the MSSM, JHEP 05 (2017) 072 [arXiv: 1701.08778$]$ [INSPIRE].

[21] L. Roszkowski, E.M. Sessolo and A.J. Williams, What next for the CMSSM and the NUHM: Improved prospects for superpartner and dark matter detection, JHEP 08 (2014) 067 [arXiv: 1405.4289] [INSPIRE].

[22] O. Buchmueller et al., The CMSSM and NUHM1 after LHC Run 1, Eur. Phys. J. C 74 (2014) 2922 [arXiv: 1312.5250] [INSPIRE].

[23] C. Strege, G. Bertone, F. Feroz, M. Fornasa, R. Ruiz de Austri and R. Trotta, Global Fits of the CMSSM and NUHM including the LHC Higgs discovery and new XENON100 constraints, JCAP 04 (2013) 013 [arXiv: 1212.2636] [INSPIRE].

[24] O. Buchmueller et al., The CMSSM and NUHM1 in Light of $7 \mathrm{TeV} L H C, B_{s} \rightarrow \mu^{+} \mu^{-}$and XENON100 Data, Eur. Phys. J. C 72 (2012) 2243 [arXiv:1207.7315] [INSPIRE].

[25] J. Chakrabortty, S. Mohanty and S. Rao, Non-universal gaugino mass GUT models in the light of dark matter and LHC constraints, JHEP 02 (2014) 074 [arXiv:1310.3620] [INSPIRE].

[26] M.A. Ajaib, SU(5) with nonuniversal gaugino masses, Int. J. Mod. Phys. A 33 (2018) 1850032 [arXiv: 1711.02560 ] [INSPIRE].

[27] J. Chakrabortty, A. Choudhury and S. Mondal, Non-universal Gaugino mass models under the lamppost of muon $(g-2)$, JHEP 07 (2015) 038 [arXiv:1503.08703] [INSPIRE].

[28] S.P. Martin, Compressed supersymmetry and natural neutralino dark matter from top squark-mediated annihilation to top quarks, Phys. Rev. D 75 (2007) 115005 [hep-ph/0703097] [INSPIRE].

[29] J. Ellis, J.L. Evans, F. Luo, K.A. Olive and J. Zheng, Stop Coannihilation in the CMSSM and SubGUT Models, Eur. Phys. J. C 78 (2018) 425 [arXiv:1801.09855] [INSPIRE].

[30] J. Ellis, F. Luo, K.A. Olive and P. Sandick, The Higgs Mass beyond the CMSSM, Eur. Phys. J. C 73 (2013) 2403 [arXiv:1212.4476] [inSPIRE].

[31] J. Ellis, J.L. Evans, A. Mustafayev, N. Nagata and K.A. Olive, The Super-GUT CMSSM Revisited, Eur. Phys. J. C 76 (2016) 592 [arXiv: 1608.05370] [INSPIRE].

[32] F. Wang, Analytical Soft SUSY Spectrum in Mirage-Type Mediation Scenarios, JHEP 11 (2018) 062 [arXiv: 1808. 08529] [inSPIRE].

[33] L. Aparicio et al., Non-thermal CMSSM with a 125 GeV Higgs, JHEP 05 (2015) 098 [arXiv: 1502.05672] [INSPIRE]. 
[34] J.R. Ellis, C. Kounnas and D.V. Nanopoulos, No Scale Supersymmetric Guts, Nucl. Phys. B 247 (1984) 373 [INSPIRE].

[35] M. Drees, Phenomenological Consequences of $N=1$ Supergravity Theories With Nonminimal Kinetic Energy Terms for Vector Superfields, Phys. Lett. B 158 (1985) 409 [INSPIRE].

[36] J.R. Ellis, K. Enqvist, D.V. Nanopoulos and K. Tamvakis, Gaugino Masses and Grand Unification, Phys. Lett. B 155 (1985) 381 [INSPIRE].

[37] M. Drees, $N=1$ Supergravity GUTs With Noncanonical Kinetic Energy Terms, Phys. Rev. D 33 (1986) 1468 [INSPIRE].

[38] B.L. Kaufman, B.D. Nelson and M.K. Gaillard, Mirage models confront the LHC: Kähler-stabilized heterotic string theory, Phys. Rev. D 88 (2013) 025003 [arXiv:1303.6575] [INSPIRE].

[39] H. Abe, T. Higaki and T. Kobayashi, KKLT type models with moduli-mixing superpotential, Phys. Rev. D 73 (2006) 046005 [hep-th/0511160] [InSPIRE].

[40] R. Blumenhagen, B. Körs, D. Lüst and S. Stieberger, Four-dimensional String Compactifications with D-branes, Orientifolds and Fluxes, Phys. Rept. 445 (2007) 1 [hep-th/0610327] [INSPIRE].

[41] K. Sumita, Nonuniversal gaugino masses in a magnetized toroidal compactification of SYM theories, JHEP 10 (2015) 156 [arXiv:1507.04408] [INSPIRE].

[42] J.E. Younkin and S.P. Martin, Non-universal gaugino masses, the supersymmetric little hierarchy problem and dark matter, Phys. Rev. D 85 (2012) 055028 [arXiv:1201.2989] [INSPIRE].

[43] S. Akula and P. Nath, Gluino-driven radiative breaking, Higgs boson mass, muon $g-2$ and the Higgs diphoton decay in supergravity unification, Phys. Rev. D 87 (2013) 115022 [arXiv:1304.5526] [INSPIRE].

[44] G. Anderson, H. Baer, C.-h. Chen and X. Tata, The Reach of Fermilab Tevatron upgrades for SU(5) supergravity models with nonuniversal gaugino masses, Phys. Rev. D 61 (2000) 095005 [hep-ph/9903370] [INSPIRE].

[45] N. Chamoun, C.-S. Huang, C. Liu and X.-H. Wu, Nonuniversal gaugino masses in supersymmetric SO(10), Nucl. Phys. B 624 (2002) 81 [hep-ph/0110332] [InSPIRE].

[46] J. Chakrabortty and A. Raychaudhuri, A Note on dimension-5 operators in GUTs and their impact, Phys. Lett. B 673 (2009) 57 [arXiv:0812.2783] [INSPIRE].

[47] S.P. Martin, Non-universal gaugino masses from non-singlet F-terms in non-minimal unified models, Phys. Rev. D 79 (2009) 095019 [arXiv: 0903.3568] [INSPIRE].

[48] S. Bhattacharya and J. Chakrabortty, Gaugino mass non-universality in an $\mathrm{SO}(10)$ supersymmetric Grand Unified Theory: Low-energy spectra and collider signals, Phys. Rev. D 81 (2010) 015007 [arXiv: 0903.4196] [INSPIRE].

[49] D. Feldman, Z. Liu and P. Nath, Gluino NLSP, Dark Matter via Gluino Coannihilation and LHC Signatures, Phys. Rev. D 80 (2009) 015007 [arXiv:0905.1148] [InSPIRE].

[50] N. Chamoun, C.-S. Huang, C. Liu and X.-H. Wu, Intermediate Scale Dependence of Non-Universal Gaugino Masses in Supersymmetric SO(10), J. Phys. G 37 (2010) 105016 [arXiv: 0909.2374] [INSPIRE].

[51] S.P. Martin, Nonuniversal gaugino masses and seminatural supersymmetry in view of the Higgs boson discovery, Phys. Rev. D 89 (2014) 035011 [arXiv:1312.0582] [INSPIRE]. 
[52] J. Kawamura and Y. Omura, Constraints on nonuniversal gaugino mass scenario using the latest LHC data, Phys. Rev. D 93 (2016) 055019 [arXiv: 1601.03484] [INSPIRE].

[53] S. Mohanty, S. Rao and D.P. Roy, Reconciling the muon $g-2$ and dark matter relic density with the LHC results in nonuniversal gaugino mass models, JHEP 09 (2013) 027 [arXiv: 1303.5830] [INSPIRE].

[54] K. Kowalska, L. Roszkowski, E.M. Sessolo and A.J. Williams, GUT-inspired SUSY and the muon g-2 anomaly: prospects for LHC 14 TeV, JHEP 06 (2015) 020 [arXiv:1503.08219] [INSPIRE].

[55] A.S. Belyaev, S.F. King and P.B. Schaefers, Muon $g-2$ and dark matter suggest nonuniversal gaugino masses: $\mathrm{SU}(5) \times A_{4}$ case study at the LHC, Phys. Rev. D 97 (2018) 115002 [arXiv: 1801.00514] [INSPIRE].

[56] J. Kawamura and Y. Omura, Study of dark matter physics in non-universal gaugino mass scenario, JHEP 08 (2017) 072 [arXiv: 1703.10379] [INSPIRE].

[57] M. Chakraborti, U. Chattopadhyay, S. Rao and D.P. Roy, Higgsino Dark Matter in Nonuniversal Gaugino Mass Models, Phys. Rev. D 91 (2015) 035022 [arXiv:1411.4517] [INSPIRE].

[58] U. Chattopadhyay, D. Das and D.P. Roy, Mixed Neutralino Dark Matter in Nonuniversal Gaugino Mass Models, Phys. Rev. D 79 (2009) 095013 [arXiv:0902.4568] [InSPIRE].

[59] D.G. Cerdeno and C. Muñoz, Neutralino dark matter in supergravity theories with non-universal scalar and gaugino masses, JHEP 10 (2004) 015 [hep-ph/0405057] [INSPIRE].

[60] U. Chattopadhyay and D.P. Roy, Higgsino dark matter in a SUGRA model with nonuniversal gaugino masses, Phys. Rev. D 68 (2003) 033010 [hep-ph/0304108] [INSPIRE].

[61] A. Corsetti and P. Nath, Gaugino mass nonuniversality and dark matter in SUGRA, strings and D-brane models, Phys. Rev. D 64 (2001) 125010 [hep-ph/0003186] [INSPIRE].

[62] S.F. King, J.P. Roberts and D.P. Roy, Natural dark matter in SUSY GUTs with non-universal gaugino masses, JHEP 10 (2007) 106 [arXiv:0705.4219] [INSPIRE].

[63] C. Balázs, T. Li, D.V. Nanopoulos and F. Wang, Supersymmetry Breaking Scalar Masses and Trilinear Soft Terms in Generalized Minimal Supergravity, JHEP 09 (2010) 003 [arXiv: 1006.5559] [INSPIRE].

[64] C. Balázs, T. Li, D.V. Nanopoulos and F. Wang, Realistic Standard Model Fermion Mass Relations in Generalized Minimal Supergravity (GmSUGRA), JHEP 02 (2011) 096 [arXiv:1101.5423] [INSPIRE].

[65] T. Li and D.V. Nanopoulos, Generalizing Minimal Supergravity, Phys. Lett. B 692 (2010) 121 [arXiv: 1002.4183] [INSPIRE].

[66] F. Wang, Supersymmetry Breaking Scalar Masses and Trilinear Soft Terms From High-Dimensional Operators in $E_{6}$ SUSY GUT, Nucl. Phys. B 851 (2011) 104 [arXiv: 1103.0069] [INSPIRE].

[67] T. Li and S. Raza, Electroweak supersymmetry from the generalized minimal supergravity model in the MSSM, Phys. Rev. D 91 (2015) 055016 [arXiv:1409.3930] [InSPIRE].

[68] T. Cheng, J. Li, T. Li, D.V. Nanopoulos and C. Tong, Electroweak Supersymmetry around the Electroweak Scale, Eur. Phys. J. C 73 (2013) 2322 [arXiv:1202.6088] [INSPIRE].

[69] F. Wang, W. Wang and J.M. Yang, A split SUSY model from SUSY GUT, JHEP 03 (2015) 050 [arXiv: 1501.02906] [InSPIRE]. 
[70] F. Wang and Y.-X. Li, Generalized Froggatt-Nielsen Mechanism, Eur. Phys. J. C 71 (2011) 1803 [arXiv: 1103.6017] [INSPIRE].

[71] F. Wang, W. Wang and J.M. Yang, Reconcile muon g-2 anomaly with LHC data in SUGRA with generalized gravity mediation, JHEP 06 (2015) 079 [arXiv:1504.00505] [INSPIRE].

[72] U. Ellwanger, J.F. Gunion and C. Hugonie, NMHDECAY: A Fortran code for the Higgs masses, couplings and decay widths in the NMSSM, JHEP 02 (2005) 066 [hep-ph/0406215] [INSPIRE].

[73] U. Ellwanger and C. Hugonie, NMHDECAY 2.0: An Updated program for sparticle masses, Higgs masses, couplings and decay widths in the NMSSM, Comput. Phys. Commun. $\mathbf{1 7 5}$ (2006) 290 [hep-ph/0508022] [INSPIRE].

[74] U. Ellwanger and C. Hugonie, NMSPEC: A Fortran code for the sparticle and Higgs masses in the NMSSM with GUT scale boundary conditions, Comput. Phys. Commun. 177 (2007) 399 [hep-ph/0612134] [INSPIRE].

[75] P.H. Chankowski, S. Pokorski and J. Rosiek, Complete on-shell renormalization scheme for the minimal supersymmetric Higgs sector, Nucl. Phys. B 423 (1994) 437 [hep-ph/9303309] [INSPIRE].

[76] A. Dabelstein, The One loop renormalization of the MSSM Higgs sector and its application to the neutral scalar Higgs masses, Z. Phys. C 67 (1995) 495 [hep-ph/9409375] [InSPIRE].

[77] D.M. Pierce, J.A. Bagger, K.T. Matchev and R.-j. Zhang, Precision corrections in the minimal supersymmetric standard model, Nucl. Phys. B 491 (1997) 3 [hep-ph/9606211] [INSPIRE].

[78] M. Frank, T. Hahn, S. Heinemeyer, W. Hollik, H. Rzehak and G. Weiglein, The Higgs Boson Masses and Mixings of the Complex MSSM in the Feynman-Diagrammatic Approach, JHEP 02 (2007) 047 [hep-ph/0611326] [INSPIRE].

[79] S. Paßehr and G. Weiglein, Two-loop top and bottom Yukawa corrections to the Higgs-boson masses in the complex MSSM, Eur. Phys. J. C 78 (2018) 222 [arXiv:1705.07909] [INSPIRE].

[80] W. Hollik and S. Paßehr, Higgs boson masses and mixings in the complex MSSM with two-loop top-Yukawa-coupling corrections, JHEP 10 (2014) 171 [arXiv:1409.1687] [INSPIRE].

[81] S. Heinemeyer, W. Hollik, H. Rzehak and G. Weiglein, The Higgs sector of the complex MSSM at two-loop order: QCD contributions, Phys. Lett. B 652 (2007) 300 [arXiv:0705.0746] [INSPIRE].

[82] S.P. Martin, Two-loop scalar self-energies and pole masses in a general renormalizable theory with massless gauge bosons, Phys. Rev. D 71 (2005) 116004 [hep-ph/0502168] [INSPIRE].

[83] S. Heinemeyer, W. Hollik, H. Rzehak and G. Weiglein, High-precision predictions for the MSSM Higgs sector at $O\left(\alpha_{b} \alpha_{s}\right)$, Eur. Phys. J. C 39 (2005) 465 [hep-ph/0411114] [INSPIRE].

[84] S.P. Martin, Strong and Yukawa two-loop contributions to Higgs scalar boson self-energies and pole masses in supersymmetry, Phys. Rev. D 71 (2005) 016012 [hep-ph/0405022] [INSPIRE]. 
[85] S.P. Martin, Two loop scalar self energies in a general renormalizable theory at leading order in gauge couplings, Phys. Rev. D 70 (2004) 016005 [hep-ph/0312092] [INSPIRE].

[86] A. Dedes, G. Degrassi and P. Slavich, On the two loop Yukawa corrections to the MSSM Higgs boson masses at large tan beta, Nucl. Phys. B 672 (2003) 144 [hep-ph/0305127] [INSPIRE].

[87] S.P. Martin, Complete two loop effective potential approximation to the lightest Higgs scalar boson mass in supersymmetry, Phys. Rev. D 67 (2003) 095012 [hep-ph/0211366] [INSPIRE].

[88] S.P. Martin, Two Loop Effective Potential for the Minimal Supersymmetric Standard Model, Phys. Rev. D 66 (2002) 096001 [hep-ph/0206136] [INSPIRE].

[89] A. Brignole, G. Degrassi, P. Slavich and F. Zwirner, On the two loop sbottom corrections to the neutral Higgs boson masses in the MSSM, Nucl. Phys. B 643 (2002) 79 [hep-ph/0206101] [INSPIRE].

[90] A. Brignole, G. Degrassi, P. Slavich and F. Zwirner, On the $O\left(\alpha_{t}^{2}\right)$ two loop corrections to the neutral Higgs boson masses in the MSSM, Nucl. Phys. B 631 (2002) 195 [hep-ph/0112177] [INSPIRE].

[91] S.P. Martin, Two loop effective potential for a general renormalizable theory and softly broken supersymmetry, Phys. Rev. D 65 (2002) 116003 [hep-ph/0111209] [INSPIRE].

[92] G. Degrassi, P. Slavich and F. Zwirner, On the neutral Higgs boson masses in the MSSM for arbitrary stop mixing, Nucl. Phys. B 611 (2001) 403 [hep-ph/0105096] [INSPIRE].

[93] J.R. Espinosa and R.-J. Zhang, Complete two loop dominant corrections to the mass of the lightest CP even Higgs boson in the minimal supersymmetric standard model, Nucl. Phys. B 586 (2000) 3 [hep-ph/0003246] [INSPIRE].

[94] J.R. Espinosa and R.-J. Zhang, MSSM lightest CP even Higgs boson mass to $O\left(\alpha_{s}\right.$ alpha(t)): The Effective potential approach, JHEP 03 (2000) 026 [hep-ph/9912236] [INSPIRE].

[95] S. Heinemeyer, W. Hollik and G. Weiglein, The Mass of the lightest MSSM Higgs boson: A Compact analytical expression at the two loop level, Phys. Lett. B 455 (1999) 179 [hep-ph/9903404] [INSPIRE].

[96] S. Heinemeyer, W. Hollik and G. Weiglein, The Masses of the neutral CP-even Higgs bosons in the MSSM: Accurate analysis at the two loop level, Eur. Phys. J. C 9 (1999) 343 [hep-ph/9812472] [INSPIRE].

[97] R.-J. Zhang, Two loop effective potential calculation of the lightest CP even Higgs boson mass in the MSSM, Phys. Lett. B 447 (1999) 89 [hep-ph/9808299] [INSPIRE].

[98] S. Heinemeyer, W. Hollik and G. Weiglein, Precise prediction for the mass of the lightest Higgs boson in the MSSM, Phys. Lett. B 440 (1998) 296 [hep-ph/9807423] [INSPIRE].

[99] S. Heinemeyer, W. Hollik and G. Weiglein, QCD corrections to the masses of the neutral CP-even Higgs bosons in the MSSM, Phys. Rev. D 58 (1998) 091701 [hep-ph/9803277] [INSPIRE].

[100] M. Carena, M. Quirós and C.E.M. Wagner, Effective potential methods and the Higgs mass spectrum in the MSSM, Nucl. Phys. B 461 (1996) 407 [hep-ph/9508343] [INSPIRE].

[101] J.A. Casas, J.R. Espinosa, M. Quirós and A. Riotto, The Lightest Higgs boson mass in the minimal supersymmetric standard model, Nucl. Phys. B 436 (1995) 3 [Erratum ibid. B 439 (1995) 466] [hep-ph/9407389] [INSPIRE]. 
[102] R. Hempfling and A.H. Hoang, Two loop radiative corrections to the upper limit of the lightest Higgs boson mass in the minimal supersymmetric model, Phys. Lett. B 331 (1994) 99 [hep-ph/9401219] [INSPIRE].

[103] P. Kant, R.V. Harlander, L. Mihaila and M. Steinhauser, Light MSSM Higgs boson mass to three-loop accuracy, JHEP 08 (2010) 104 [arXiv: 1005.5709] [INSPIRE].

[104] R.V. Harlander, P. Kant, L. Mihaila and M. Steinhauser, Higgs boson mass in supersymmetry to three loops, Phys. Rev. Lett. 100 (2008) 191602 [arXiv:0803.0672] [INSPIRE].

[105] S.P. Martin, Three-loop corrections to the lightest Higgs scalar boson mass in supersymmetry, Phys. Rev. D 75 (2007) 055005 [hep-ph/0701051] [InSPIRE].

[106] E. Bagnaschi, J. Pardo Vega and P. Slavich, Improved determination of the Higgs mass in the MSSM with heavy superpartners, Eur. Phys. J. C 77 (2017) 334 [arXiv:1703.08166] [INSPIRE].

[107] G. Lee and C.E.M. Wagner, Higgs bosons in heavy supersymmetry with an intermediate $m_{A}$, Phys. Rev. D 92 (2015) 075032 [arXiv: 1508.00576] [INSPIRE].

[108] J. Pardo Vega and G. Villadoro, SusyHD: Higgs mass Determination in Supersymmetry, JHEP 07 (2015) 159 [arXiv: 1504.05200] [INSPIRE].

[109] E. Bagnaschi, G.F. Giudice, P. Slavich and A. Strumia, Higgs Mass and Unnatural Supersymmetry, JHEP 09 (2014) 092 [arXiv: 1407.4081] [INSPIRE].

[110] P. Draper, G. Lee and C.E.M. Wagner, Precise estimates of the Higgs mass in heavy supersymmetry, Phys. Rev. D 89 (2014) 055023 [arXiv: 1312.5743] [InSPIRE].

[111] G.F. Giudice and A. Strumia, Probing High-Scale and Split Supersymmetry with Higgs Mass Measurements, Nucl. Phys. B 858 (2012) 63 [arXiv:1108.6077] [INSPIRE].

[112] R.V. Harlander, J. Klappert, A.D. Ochoa Franco and A. Voigt, The light CP-even MSSM Higgs mass resummed to fourth logarithmic order, Eur. Phys. J. C $\mathbf{7 8}$ (2018) 874 [arXiv: 1807.03509] [INSPIRE].

[113] H. Bahl and W. Hollik, Precise prediction of the MSSM Higgs boson masses for low $M_{A}$, JHEP 07 (2018) 182 [arXiv:1805.00867] [INSPIRE].

[114] P. Athron et al., FlexibleSUSY 2.0: Extensions to investigate the phenomenology of SUSY and non-SUSY models, Comput. Phys. Commun. 230 (2018) 145 [arXiv:1710.03760] [INSPIRE].

[115] H. Bahl, S. Heinemeyer, W. Hollik and G. Weiglein, Reconciling EFT and hybrid calculations of the light MSSM Higgs-boson mass, Eur. Phys. J. C 78 (2018) 57 [arXiv: 1706.00346] [INSPIRE].

[116] F. Staub and W. Porod, Improved predictions for intermediate and heavy Supersymmetry in the MSSM and beyond, Eur. Phys. J. C 77 (2017) 338 [arXiv:1703.03267] [InSPIRE].

[117] P. Athron, J.-h. Park, T. Steudtner, D. Stöckinger and A. Voigt, Precise Higgs mass calculations in (non-)minimal supersymmetry at both high and low scales, JHEP 01 (2017) 079 [arXiv: 1609.00371] [INSPIRE].

[118] H. Bahl and W. Hollik, Precise prediction for the light MSSM Higgs boson mass combining effective field theory and fixed-order calculations, Eur. Phys. J. C 76 (2016) 499 [arXiv: 1608.01880] [INSPIRE]. 
[119] T. Hahn, S. Heinemeyer, W. Hollik, H. Rzehak and G. Weiglein, High-Precision Predictions for the Light CP -Even Higgs Boson Mass of the Minimal Supersymmetric Standard Model, Phys. Rev. Lett. 112 (2014) 141801 [arXiv:1312.4937] [INSPIRE].

[120] S. Heinemeyer, W. Hollik and G. Weiglein, FeynHiggs: A Program for the calculation of the masses of the neutral CP even Higgs bosons in the MSSM, Comput. Phys. Commun. 124 (2000) 76 [hep-ph/9812320] [INSPIRE].

[121] T. Hahn, S. Heinemeyer, W. Hollik, H. Rzehak and G. Weiglein, FeynHiggs: A program for the calculation of MSSM Higgs-boson observables - Version 2.6.5, Comput. Phys. Commun. 180 (2009) 1426 [INSPIRE].

[122] G. Degrassi, S. Heinemeyer, W. Hollik, P. Slavich and G. Weiglein, Towards high precision predictions for the MSSM Higgs sector, Eur. Phys. J. C 28 (2003) 133 [hep-ph/0212020] [INSPIRE].

[123] ATLAS collaboration, Measurements of the Higgs boson production and decay rates and coupling strengths using pp collision data at $\sqrt{s}=7$ and $8 \mathrm{TeV}$ in the ATLAS experiment, ATLAS-CONF-2015-007.

[124] CMS collaboration, Precise determination of the mass of the Higgs boson and tests of compatibility of its couplings with the standard model predictions using proton collisions at 7 and 8 TeV, Eur. Phys. J. C 75 (2015) 212 [arXiv:1412.8662] [INSPIRE].

[125] J. Cao, Y. He, P. Wu, M. Zhang and J. Zhu, Higgs Phenomenology in the Minimal Dilaton Model after Run I of the LHC, JHEP 01 (2014) 150 [arXiv: 1311.6661] [INSPIRE].

[126] J. Cao, F. Ding, C. Han, J.M. Yang and J. Zhu, A light Higgs scalar in the NMSSM confronted with the latest LHC Higgs data, JHEP 11 (2013) 018 [arXiv:1309.4939] [INSPIRE].

[127] P. Bechtle et al., HiggsBounds-4: Improved Tests of Extended Higgs Sectors against Exclusion Bounds from LEP, the Tevatron and the LHC, Eur. Phys. J. C 74 (2014) 2693 [arXiv:1311.0055] [INSPIRE].

[128] ATLAS collaboration, Search for the direct production of charginos and neutralinos in final states with tau leptons in $\sqrt{s}=13$ TeV pp collisions with the ATLAS detector, Eur. Phys.

J. C 78 (2018) 154 [arXiv: 1708.07875] [InSPIRE].

[129] BABAR collaboration, J.P. Lees et al., Precision Measurement of the $B \rightarrow X_{s} \gamma$ Photon Energy Spectrum, Branching Fraction and Direct CP Asymmetry $A_{C P}\left(B \rightarrow X_{s+d} \gamma\right)$, Phys. Rev. Lett. 109 (2012) 191801 [arXiv:1207.2690] [INSPIRE].

[130] BABAR collaboration, J.P. Lees et al., Evidence for an excess of $\bar{B} \rightarrow D^{(*)} \tau^{-} \bar{\nu}_{\tau}$ decays, Phys. Rev. Lett. 109 (2012) 101802 [arXiv:1205.5442] [INSPIRE].

[131] LHCb collaboration, First Evidence for the Decay $B_{s}^{0} \rightarrow \mu^{+} \mu^{-}$, Phys. Rev. Lett. 110 (2013) 021801 [arXiv: 1211.2674] [INSPIRE].

[132] Particle Data Group collaboration, C. Patrignani et al., Review of Particle Physics, Chin. Phys. C 40 (2016) 100001 [InSPIRE].

[133] F. Jegerlehner, Essentials of the Muon g-2, Acta Phys. Polon. B 38 (2007) 3021 [hep-ph/0703125] [INSPIRE].

[134] J. Bijnens and J. Prades, The Hadronic Light-by-Light Contribution to the Muon Anomalous Magnetic Moment: Where do we stand?, Mod. Phys. Lett. A 22 (2007) 767 [hep-ph/0702170] [INSPIRE]. 
[135] S. Heinemeyer, D. Stöckinger and G. Weiglein, Electroweak and supersymmetric two-loop corrections to $(g-2)_{\mu}$, Nucl. Phys. B 699 (2004) 103 [hep-ph/0405255] [INSPIRE].

[136] A. Czarnecki, W.J. Marciano and A. Vainshtein, Refinements in electroweak contributions to the muon anomalous magnetic moment, Phys. Rev. D 67 (2003) 073006 [Erratum ibid. D 73 (2006) 119901] [hep-ph/0212229] [INSPIRE].

[137] D. Stöckinger, The Muon Magnetic Moment and Supersymmetry, J. Phys. G 34 (2007) R45 [hep-ph/0609168] [INSPIRE].

[138] A. Arhrib and S. Baek, Two loop Barr-Zee type contributions to $(g-2)_{\mu}$ in the MSSM, Phys. Rev. D 65 (2002) 075002 [hep-ph/0104225] [inSPIRE].

[139] K.-m. Cheung, C.-H. Chou and O.C.W. Kong, Muon anomalous magnetic moment, two Higgs doublet model and supersymmetry, Phys. Rev. D 64 (2001) 111301 [hep-ph/0103183] [INSPIRE].

[140] E. Bagnaschi et al., Likelihood Analysis of the pMSSM11 in Light of LHC 13-TeV Data, Eur. Phys. J. C 78 (2018) 256 [arXiv:1710.11091] [InSPIRE].

[141] P. Athron et al., GM2Calc: Precise MSSM prediction for $(g-2)$ of the muon, Eur. Phys. J. C 76 (2016) 62 [arXiv:1510.08071] [INSPIRE].

[142] Planck collaboration, P.A.R. Ade et al., Planck 2013 results. XVI. Cosmological parameters, Astron. Astrophys. 571 (2014) A16 [arXiv:1303.5076] [InSPIRE].

[143] WMAP collaboration, G. Hinshaw et al., Nine-Year Wilkinson Microwave Anisotropy Probe (WMAP) Observations: Cosmological Parameter Results, Astrophys. J. Suppl. 208 (2013) 19 [arXiv:1212.5226] [inSPIRE].

[144] XENON collaboration, E. Aprile et al., Dark Matter Search Results from a One Ton-Year Exposure of XENON1T, Phys. Rev. Lett. 121 (2018) 111302 [arXiv:1805.12562] [INSPIRE].

[145] G. Bélanger, F. Boudjema, A. Pukhov and A. Semenov, MicrOMEGAs: A Tool for dark matter studies, Nuovo Cim. C 033N2 (2010) 111 [arXiv:1005.4133] [INSPIRE].

[146] G. Bélanger, F. Boudjema, A. Pukhov and A. Semenov, Dark matter direct detection rate in a generic model with MicrOMEGAs 2.2, Comput. Phys. Commun. 180 (2009) 747 [arXiv: 0803.2360] [INSPIRE].

[147] G. Bélanger, F. Boudjema, A. Pukhov and A. Semenov, MicrOMEGAs 2.0: A Program to calculate the relic density of dark matter in a generic model, Comput. Phys. Commun. 176 (2007) 367 [hep-ph/0607059] [INSPIRE].

[148] J. Cao, X. Guo, Y. He, P. Wu and Y. Zhang, Diphoton signal of the light Higgs boson in natural NMSSM, Phys. Rev. D 95 (2017) 116001 [arXiv:1612.08522] [INSPIRE].

[149] K.J. de Vries et al., The pMSSM10 after LHC Run 1, Eur. Phys. J. C 75 (2015) 422 [arXiv: 1504.03260] [INSPIRE]. 